\title{
Review of real-time vehicle schedule recovery methods in transportation services
}

\author{
Monize Sâmara Visentini • Denis Borenstein • \\ Jing-Quan Li · Pitu B. Mirchandani
}

Received: 10 November 2011 / Accepted: 28 June 2013

(C) Springer Science+Business Media New York 2013

\begin{abstract}
This paper presents a comprehensive review on methods for real-time schedule recovery in transportation services. The survey concentrates on published research on recovery of planned schedules in the occurrence of one or several severe disruptions such as vehicle breakdowns, accidents, and delays. Only vehicle assignment and rescheduling are reviewed; crew scheduling and passenger logistics problems during disruptions are not. Real-time vehicle schedule recovery problems (RTVSRP) are classified into three classes: vehicle rescheduling, for road-based services, trainbased rescheduling, and airline schedule recovery problems. For each class, a classification of the models is presented based on problem formulations and solution strategies. The paper concludes that RTVSRP is a challenging problem that requires quick and good quality solutions to very difficult and complex situations, involving several different contexts, restrictions, and objectives. The paper also identifies research gaps to be investigated in the future, stimulating research in this area.
\end{abstract}

M. S. Visentini · D. Borenstein $(\varangle)$

Management School, Universidade Federal do Rio Grande do Sul, Porto Alegre, Brazil

e-mail: denisb@ea.ufrgs.br

M. S. Visentini

e-mail: monize.s.visentini@gmail.com

J.-Q. Li

California PATH, University of California, Berkeley,

Berkeley, CA, USA

e-mail: jingquan@path.berkeley.edu

P. B. Mirchandani

School of Computing, Informatics, and Decision Systems Engineering, Arizona State University, Phoenix, AZ, USA

e-mail: pitu@asu.edu
Keywords Rescheduling · Recovering · Vehicle · Train · Aircraft

\section{Introduction}

Many optimization-based algorithms have been developed in the last decades for generating vehicle schedules in transportation services. However, the planned schedules are sometimes disrupted by unforeseen events. Some of these disruptions are severe enough to prevent the system from operating as planned, such as inclement weather, terrorist events, and vehicle breakdowns. When these situations occur, rescheduling is approached as a real-time vehicle schedule recovery problem (RTVSRP). The main objective of this paper is to review and synthesize the literature on contributions toward solving such problems for ground and airline transportation services.

Clausen et al. (2010) classify a disrupted situation where the deviation from the plan is sufficiently large to impose a substantial change in operations. Severe weather conditions, accidents, maintenance, and the breakdown of vehicles are the examples of possible disruptions that demand the rescheduling of vehicles. Disruption data in transportation services are impressive. In 2008, on average $17.67 \%$ of the Brazilian commercial flights were delayed more than $30 \mathrm{~min}$, and on average $2.67 \%$ were canceled according to Brazilian Civil Aviation Agency (2010). Similar numbers are presented by Eggenberg et al. (2010) for European flights. In the railway sector, Jespersen-Groth et al. (2009) reported that the Dutch railway network has approximately 17 disruptions per day with an average duration of $1.8 \mathrm{~h}, 35 \%$ of them being related to technical failures and $35 \%$ related to the third parties (e.g., accidents). For transit service examples, San Francisco Municipal Transportation Agency had 
only $71 \%$ bus trips on time from 2007 to 2010 (see http:// www.sfog.us/sfmuni.htm). SunTran transit agency in Tucson, Arizona, had 82 road calls in July 2011 (see SunTran 2011), where small accidents were fixed quickly by drivers, but some serious accidents led to towing the disabled bus back to a depot.

The economic impact of disruptions is also significant. Disruptions introduce additional costs and decrease in service level. Based on data from the Air Transportation of America, Ball et al. (2007) reported that US\$ 6.5 billion were spent in 2000 by customers and airline companies to deal with delays. According to Guarino and Firestine (2010) several snowstorms in the USA during February 2010 generated the greatest proportion of weather-related cancelations on record, with $4.2 \%$ of all flights being canceled (20,206 flights). These cancelations were estimated to have a cost of about \$80-\$100 million. Severe weather has also caused delays and cancelations in Swedish railways. During the winter of 2010-2011, heavy snowfall and record cold resulted in approximately four million hours in delays and $\$ 372$ million in lost working hours (Swedish Transport Administration 2011).

Until recently, real-time vehicle schedule recovery was exclusively conducted by human schedulers, based on their experience and common sense. However, the implementation of new information and communication technologies (e.g., automatic vehicle locaters, the global positioning system, geographical information systems, and wireless communication) in transportation systems and the unprecedented increase in the capacity of the computers to solve large instances of problems enables providing real-time information and implement real-time disruption recovery algorithms at reasonable costs. As a consequence, a growing body of contributions on several aspects of real-time vehicle schedule recovery in transportation services has appeared in the operations research and related literature. Indeed, some of the recently proposed models present increased levels of realism and incorporate a large variety of needed constraints and operational requirements that have resulted in effective computer algorithms used by transportation/logistic companies.

An interesting alternative to address disruption problems is to build robustness into the schedule throughout schedule design process. The main idea of robust schedule is to incorporate the possibility of disruptions during the schedule design to enhance potential recovery actions, such as adding buffers or slack time between operations in schedules or having standby vehicles and part-time crews. Ahuja et al. (2009) present a compilation of papers dealing with robust optimization theory in transportation systems. Robust scheduling is becoming an important topic in transportation services and several research papers have appeared in the last two decades on this subject. Huisman et al. (2004), Kramkowski et al. (2009), and Amberg et al. (2012) deal with robust bus scheduling; Zwaneveld et al. (1996), Zwaneveld et al. (2001), Fischetti et al. (2009), and Caprara et al. (2010) consider robust train scheduling; Ageeva (2000), Lan et al. (2006), Lee et al. (2007), Weide et al. (2009), Borndörfer et al. (2009), and Dück et al. (2012) address robust aircraft scheduling. As pointed out by Ahuja et al. (2009) and Clausen et al. (2010), robust scheduling and real-time vehicle schedule recovery are tightly coupled problems. The former constitutes a proactive approach, while the latter assumes a reactive approach to deal with disruptions during operation. This paper is focused on the real-time vehicle schedule recovery problem.

Another related aspect in the rescheduling context is stability. This term refers to schedules where "there is little deviation between the pre-[initial] schedule and the executed schedule" (Leus and Herroelen 2005). Stability should be an important consideration in the schedule design so that during rescheduling, perturbations in the initial scheduling are small (Rangsaritratsamee et al. 2004), so that the costs involved in the disruption management process are also small. In the transportation context, stability can be a schedule characteristic that relates to the ability to return to normal operation after a disturbance occurs (D'Ariano 2008). Although this term is often used in production management as a rescheduling performance index (Herroelen and Leus 2004; Raheja and Subramaniam 2002), stability has not been a major consideration due to the complex dynamics of the disruption management in transportation.

From a modeling point of view, the RTVSRP is modeled and solved using similar models, but not the same, as their offline planning counterparts. In general, an underlying network structure representing the problem is designed. This network representation describes how vehicles can be rescheduled, taking into consideration the current planned schedule, the current situation when the disruption occurred, and technical and timing constraints. Based on the network representation, optimization- and heuristic-based methods are developed. The most important modeling difference between the realtime recovery problem and the schedule planning problem is that the underlying network is dependent on the existing situation and feasible alternatives to address the disruption. As the number of alternatives increases, the complexity of the problem increases exponentially. Clearly, the RTVSRP also differs with the industry. In the airline industry, the problem is characterized by the high costs involved and the highly regulated environment. In bus public transportation services, the number of alternatives to recover the disruptions is extremely large when the transportation systems of large cities are considered. In the railway sector, the sharing of some resources (tracks) is critical in its operations, and additional technical constraints demand the introduction of several new features in the modeling approaches. However, schedule recovery requires solutions to be provided very quickly. The main challenge for the scheduling researchers is to develop pow- 
erful and robust algorithms for quickly solving these large and complex problems.

This paper reviews most of the recent contributions dealing with RTVSRP regarding both airline and ground transportation. We have emphasized textual description, given the heterogeneity of formulations and models presented in the literature. Most of the formulations reviewed have been proposed in the last decade. This survey mostly focuses on papers in OR-oriented journals. Also, the authors limit this survey to vehicle rescheduling. Crew scheduling and passenger logistics problems during disruption are not reviewed and even though many studies in these areas are certainly relevant to the real-time disruption management. The paper does not review vessel schedule recovery problems, since there is little reported research on this problem. An exception is the work of Dirksen (2011) which presents mathematical models for disruption in shipping inspired by developments for the aircraft schedule recovery problem. For a deeper understanding on ship routing and scheduling see the survey by Christiansen et al. (2004).

The paper is organized as follows. Section 2 discusses the general concepts for the real-time schedule recovery problem, including general definitions and network structures developed to solve the problem. Sections 3, 4, and 5 survey the vehicle rescheduling problem (VRSP) for bus/freight services, the rescheduling of train services after disruptions, and the aircraft rescheduling problems, respectively. Section 6 presents some concluding remarks, focusing on similarities and differences among road, railway, and aircraft-based transportation. The paper ends presenting some directions for further research.

\section{Basic models and problem formulations}

Real-time vehicle schedule recovery problem can be stated as follows. Given a set of depots (or stations), a set of vehicles, and a series of trips with fixed starting and ending times or with service time windows, given the travel times between all pairs of locations, given a serious disruption that interrupts at least one currently scheduled trip and given the current location and the current status of the vehicles in the system, find a feasible schedule that optimizes a set of (sometimes conflicting) objectives, in which trips are either rescheduled (some with delays) or canceled, and each vehicle performs a feasible sequence of trips (or track segments, in the case of trains). When the vehicles are buses or trucks, the problem will be referred to as vehicle rescheduling problem (VRSP). When the vehicle is a train, the problem will be called train rescheduling problem (TRP). Finally, when the vehicle is an airplane, the problem will be called aircraft rescheduling problem (ARP).
A very important aspect in the rescheduling process is the strategy used. Based on the literature (Acuna-Agost et al. 2011; Ionescu et al. 2010), it is possible to identify three rescheduling strategies: dynamic, predictive (also called offline), and reactive (also called on-line). In dynamic strategy, trains are dispatched using local information with a decentralized control method based on rules. The strategy is widely used by human schedulers, since it is easy to implement and offer solutions very quickly. Predictive schedules are generated within robust scheduling contexts, where the main objective is to generate schedules able to deal with minor disturbances. In such situation, all disruptions and scenarios are known in advance. Reactive strategies consist on finding a new schedule after the occurrence of one or several events, including severe disruptions, minimizing some measure of the effects. This strategy is based on an accurate monitoring of the resources involved, in terms of capacity, position, and speed. New schedules are generated in real-time. Ionescu et al. (2010) compared these strategies for the airline recovery problem using data from an European airline. Two criteria were used to evaluate them: punctuality of flight arrival and run-time efficiency. The dynamic strategy, as expected, offered poor solutions very quickly. They also concluded that the offline approach, although more efficient, is not a practicable recovery technique in comparison with the online strategy, although it provides a theoretical lower bound for the recovery problem.

Since all classes of RTVSRPs are strongly related to the counterpart schedule planning problems, they are usually formulated and solved using similar, but not identical network models. In these problems, the network will be referred to as the underlying recovery network. Based on such networks, mathematical formulations and solution methods are developed and tested.

The main purpose of the underlying recovery network is to represent all possible alternatives for rescheduling. The definition of the network is dependent on the pre-assigned configuration of the system when a disruption occurs, the status of the vehicles in the system, and the times needed to perform compatible sequences of trips (flight legs in the airline context; track segments in the railway context). As identified by Clausen et al. (2010), the literature on airline recovery generally uses three types of network representations; namely, connection networks (CNs), time-line networks (TLN), and time-band networks (TBN). CNs are activity-on-node networks, where nodes represent trips, stations, airports, etc., and the arcs describe connections between the nodes, representing, for example, deadheading. Each path of a source to a sink refers to a feasible sequence of a trip for a vehicle. $\mathrm{CNs}$ are widely used in planning schedules and their application to recovery problems is quite natural. Examples of CNs abound in the literature (see Freling et al. 2001; Bertsimas and Patterson 2000, and Li et al. 2004). The other 
Table 1 Hypothetical train scheduling

\begin{tabular}{lllllll}
\hline Trains & $\begin{array}{l}\text { Train } \\
\text { number }\end{array}$ & $\begin{array}{l}\text { Station of } \\
\text { origin }\end{array}$ & $\begin{array}{l}\text { Station of } \\
\text { destination }\end{array}$ & $\begin{array}{l}\text { Train } \\
\text { departure }\end{array}$ & $\begin{array}{l}\text { Train } \\
\text { arrival }\end{array}$ & $\begin{array}{l}\text { Travel } \\
\text { time }\end{array}$ \\
\hline Train A & 01 & LED & SHL & $17: 00$ & $18: 30$ & $1: 30$ \\
& 02 & SHL & LED & $19: 00$ & $20: 30$ & $1: 30$ \\
Train B & 11 & HFD & NVP & $16: 20$ & $17: 10$ & 0.50 \\
& 12 & NVP & HFD & $17: 20$ & $18: 20$ & $1: 00$ \\
& 13 & HFD & NVP & $19: 00$ & $20: 00$ & $1: 00$ \\
& 14 & NVP & HFD & $20: 15$ & $21: 15$ & $1: 00$ \\
Train C & 31 & SHL & HFD & $15: 10$ & $16: 10$ & $1: 00$ \\
& 32 & HFD & LED & $16: 30$ & $17: 10$ & $0: 40$ \\
& 33 & LED & HFD & $17: 30$ & $18: 10$ & $0: 40$ \\
& 34 & HFD & SHL & $18: 50$ & $20: 00$ & $1: 10$ \\
\hline
\end{tabular}

Fig. 1 The time-line network of the sample schedule (based on Clausen et al. 2010)

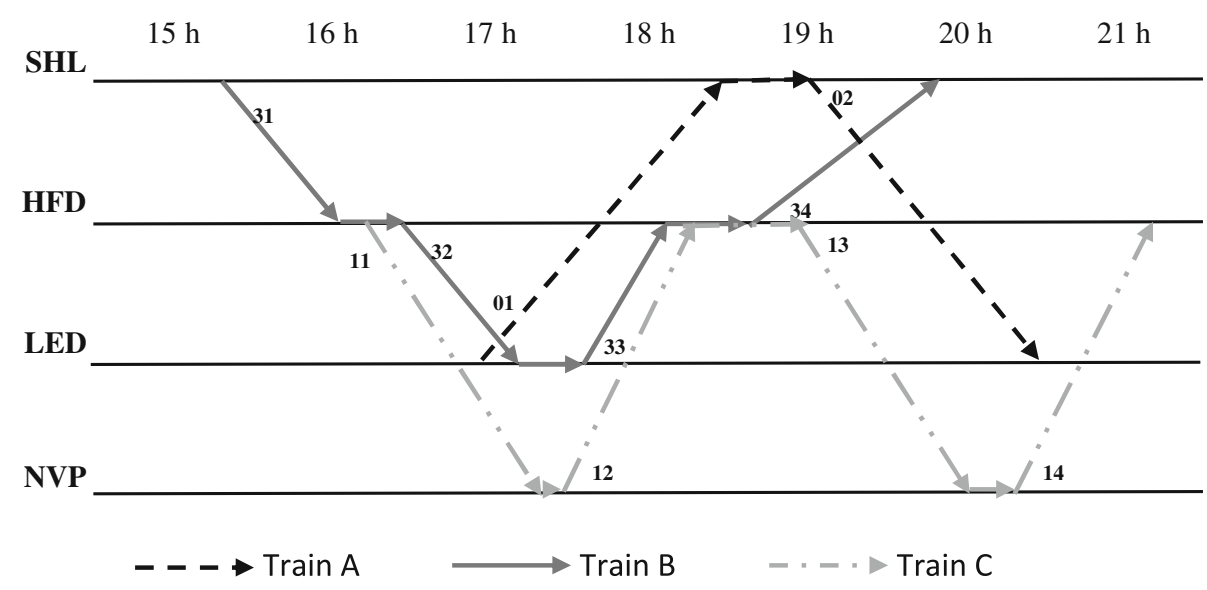

two network types have their main applications in recovery problems. $T L N$ are activity-on-edge networks, in which all events (represented in the nodes) of a resource (such as station, airport, and track segments) are placed on a timeline corresponding to that resource. Arcs connect events in the same time-line (which involves the same resource) or in different time-lines (when different resources are involved). $T B N$ were developed by Argüello et al. (1997) to specifically deal with aircraft recovery. They are networks positioned with respect to two-dimensional axes, one representing time and the other one space or stations. A node placed in this space represents specific activities at a station during a segment of time. Arcs represent arrival to or departures from a station if they are directed into a node or originated from a node, respectively. In order to illustrate these networks, we use a hypothetical example, with four Netherland train stations, as follows: Schiphol (SHL), Hoofddorp (HFD), Leiden (LED), and Niew Vennep (NVP), and three trains, which stop at each station for a period for passenger embarking and disembarking, and cleaning activities. Table 1 presents the trains' timetable. Figures 1, 2, and 3 show the TLN, TBN, and CNs, respectively, for this sample schedule. The TBN represents a network that can be constructed dynamically as disruptions occur. For the hypothetical example of Table 1, suppose that train $C$ becomes out of service from 15:00 to 22:00 for unplanned maintenance. We defined the time-bands to be around $30 \mathrm{~min}$. Figure 2 shows not all, but some of the possible new schedules for the two remaining trains, one starting in LED and the other one in HFD; and ending in either LED or HFD. In Fig. 2, an illustrative case for a recovery rotation for train B is SHL-31-11 (delayed $40 \mathrm{~min}$ ) 12 (delayed $70 \mathrm{~min}$ ) - 34 (delayed $70 \mathrm{~min}$ ) - SHL for which the complete path is SHL (source)-HFD-NVP-HFDSHL-SHL (sink).

Considering these network models, the RTVSRP has been formulated based on the classical network problem formulations such as the minimum cost flow problem, the multi-commodity network flow, and the set packing problem; by adding some binary variables, together with constraints representing problems peculiarities, and expanding the objective function to include delay and cancelation costs (Clausen et al. 2010; Törnquist 2006). The literature does not present a generic formulation for this problem, given the heterogeneity of contexts where this problem can be applied both in terms of the disruption and the attendant recovery decisions. However, based on the models available in the lit- 
Fig. 2 A partial time-band network of the sample schedule (based on Clausen et al. 2010)

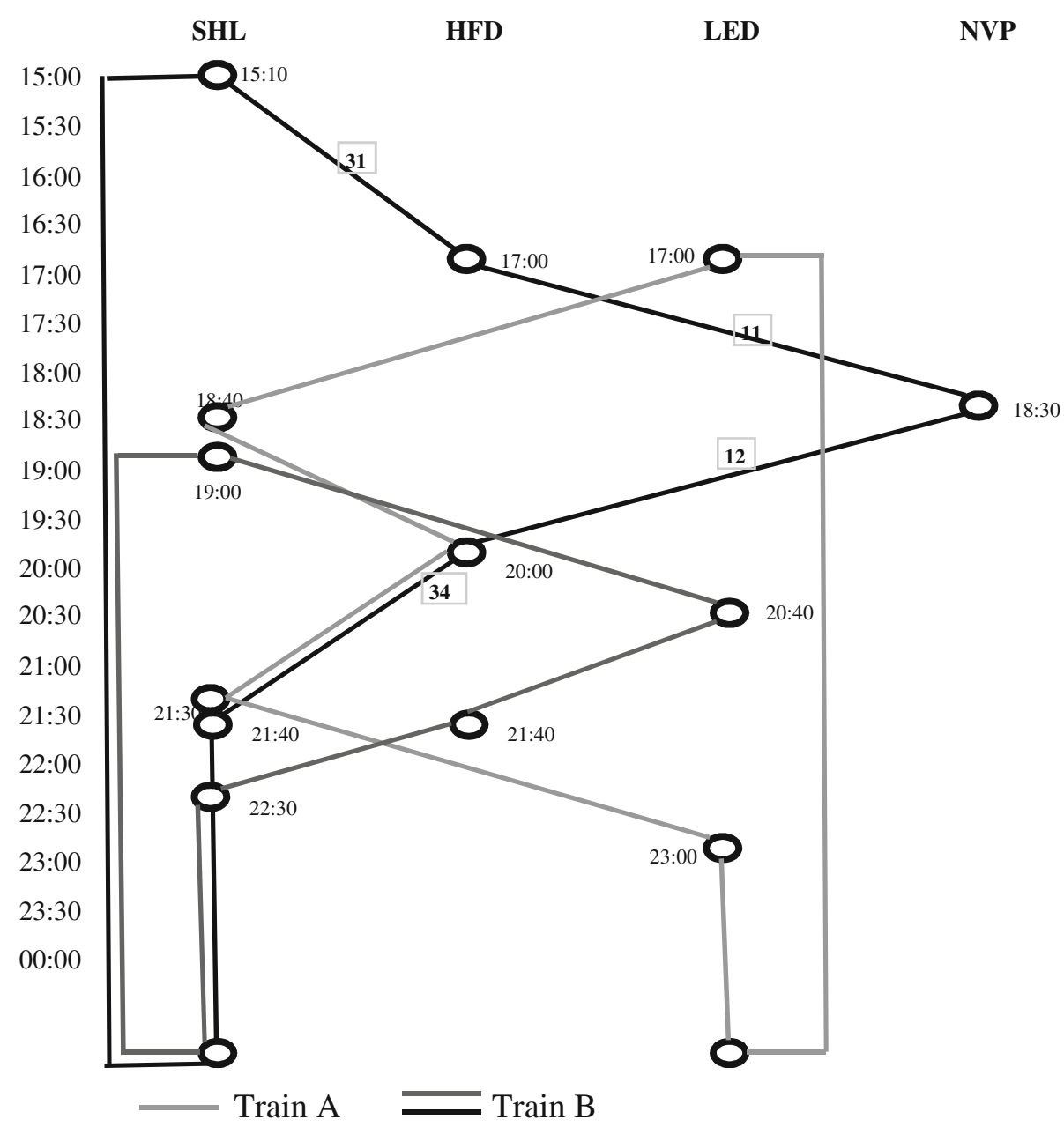

Fig. 3 The connection network of the sample schedule (based on Clausen et al. 2010)

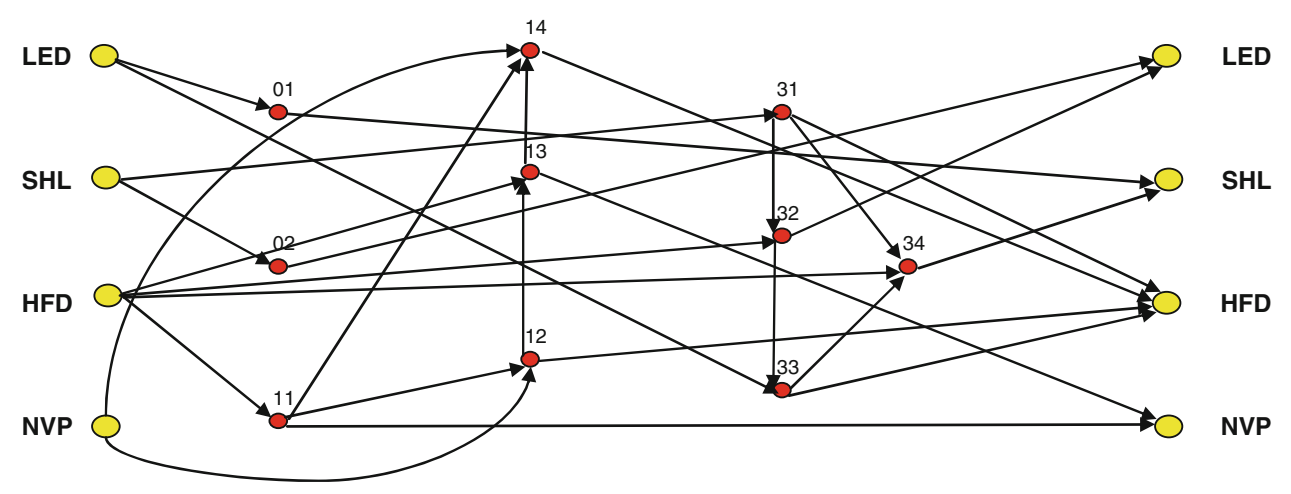

erature, it is possible to formulate a generic model for the RTVSRP based on a CN, where the nodes are represented by trips, and the arcs connect two compatible pair of trips. Trips $\mathrm{i}$ and $\mathrm{j}$ are a compatible pair of trips if the same vehicle can reach the starting point of trip $j$ after it finishes trip $i$ and the vehicle has technical and capacity attributes to perform both the trips. It should be noted that several relevant aspects found in real-world rescheduling problems are missing in this representation. Peculiarities found in the train rescheduling problem, such as the sharing of uni- or bi-directional tracks in a single or double line or the slot sharing at airports in the context of ARP, are for now intentionally excluded for the sake of generality for the three classes of problems. The main objective now for introducing the underlying $\mathrm{CN}$ is to explicitly illustrate the main constraints involved in a large fraction of instances of the problems described in the literature.

Before giving the formulation, we introduce the following notation:

$B_{\mathrm{i}}=$ prescribed starting time of trip i.

$W_{\mathrm{i}}=$ prescribed time of trip $\mathrm{i}$. 
$T_{\mathrm{ij}}=$ travel time from the ending point of trip $\mathrm{i}$ to the starting time of trip $\mathrm{j}$.

$D_{\mathrm{i}}=$ maximum delay allowed for trip i.

$U=$ service time at station.

$P_{\mathrm{i}}=$ trip i delay cost.

$C_{\mathrm{i}}=$ trip i cancelation cost.

$F=$ time of the disruption.

Let $A$ be the set of trips that are being served by vehicles at the instant of a trip disruption. Let $N$ denote the set of all future service trips, including the disrupted trip (which still needs to be served), numbered according to non-decreasing starting times. Trips in set $N$ might be re-assigned to different vehicles from their pre-assigned ones in the initial schedule, while trips in set $A$ cannot be reassigned. Set $A \cup B$ can be seen as the set of all unfinished trips. Let $Z$ denote the set of all compatible pair of trips (i, j), in which $\mathrm{j}$ starts after $F$. Assume that each vehicle $k \in K$ in the network can be rescheduled at time $F$. In reality, this number could be much smaller than the number of vehicles in the system because technical and capacity constraints, as well as the position of the vehicles in the network in time $F$, will limit this number. The model has three sets of decision variables, $s t_{i}$, the starting time of trip $\mathrm{i} \in A \cup B$ and two binary ones, namely $x_{\mathrm{ij}}^{k}$ and $z_{\mathrm{i}}$, where $x_{\mathrm{ij}}^{k}=1$ if vehicle $k \in K$ is assigned to trip $\mathrm{j} \in N$ directly after trip $\mathrm{i} \in A \cup B$, and $z_{\mathrm{i}}=1$ if service trip $\mathrm{i} \in N$ is canceled. Thus the RTVSRP can be formulated as follows:

$\operatorname{Min} \sum_{(k \in K)} \sum_{(\mathrm{i}, \mathrm{j}) \in Z} c_{\mathrm{ij}}^{k} x_{\mathrm{ij}}^{k}+\sum_{(\mathrm{i} \in N)} C_{\mathrm{i}} z_{\mathrm{i}}+\sum_{(\mathrm{i} \in N)} P_{\mathrm{i}}\left(s t_{\mathrm{i}}-B_{\mathrm{i}}\right)$

subject to

$\sum_{\mathrm{j}:(\mathrm{i}, \mathrm{j}) \in N} x_{\mathrm{ij}}^{k}=\sum_{\mathrm{j}:(\mathrm{i}, \mathrm{j}) \in N} x_{\mathrm{ji}}^{k} \quad \forall \mathrm{i} \in N, \quad k \in K$

$\sum_{(k \in K)} \sum_{\mathrm{j}:(\mathrm{i}, \mathrm{j}) \in Z} x_{\mathrm{ij}}^{k}+z_{\mathrm{i}}=1 \quad \forall \mathrm{i} \in N$

$s t_{\mathrm{i}}=B_{\mathrm{i}} \quad \forall \mathrm{i} \in A$

$s t_{\mathrm{i}} \geq B_{\mathrm{i}} \quad, \forall \mathrm{i} \in N$

$s t_{\mathrm{i}} \leq B_{\mathrm{i}}+D_{\mathrm{i}} \quad \forall \mathrm{i} \in N$

$s t_{\mathrm{j}} \geq\left(s t_{\mathrm{i}}+U+T_{\mathrm{ij}}\right) x_{\mathrm{ij}}^{k} \quad \forall(\mathrm{i}, \mathrm{j}) \in Z, \quad k \in K$

$x_{\mathrm{ij}}^{k} \in\{0,1\} \quad \forall(\mathrm{i}, \mathrm{j}) \in Z, \quad k \in K$

$s t_{\mathrm{i}} \geq 0, z_{\mathrm{i}} \in\{0,1\} \quad \forall \mathrm{i} \in A \cup B$

where $c_{\mathrm{ij}}^{k}$ is the distance-based cost of arc (i,j) by vehicle $k$ to perform trips $\mathrm{i}$ and $\mathrm{j}$. This $c_{\mathrm{ij}}^{k}$ could take into account aspects such as fixed and operation costs of vehicle $k$ (applicable mostly for airplanes and trains), distance traveled, and penalties due to rescheduling. The objective function (1) minimizes the weighted sum of operations, delay, penalty, and trip cancelation costs. Constraints (2) guarantee flow conservation for vehicles. Constraints (3) assure that any trip $i \in N$ is either serviced or canceled. Constraints (4) set the starting time of trips in $A$ as the prescribed starting time, since they cannot be rescheduled. Constraints (5) and (6) guarantee that the starting time of any trip $\mathrm{i} \in N$ is not earlier than its prescribed starting time and does not exceed its delay limit. Constraints (7) establish that the starting time of each future trip $\mathrm{j} \in N$ is dependent on the required deadheading (traveling plus unloading) times of its previous trip $\mathrm{i} \in N$. Constraints (7) are nonlinear. Li et al. (2008) has showed that this term can be easy linearized using the big-M technique. Constraints (8)-(9) define the domain of the decision variables.

The RTVRSP has also been formulated and solved based on models and methods developed for the real-time schedule recovery of machines (Raheja and Subramaniam 2002), mainly in the railway transportation. Vehicles can be viewed as jobs, and trips or block segments (in railway context) as machines. The processing of a job by a machine for a given amount of time, characterizing an operation, corresponds to the allocation of a vehicle to a trip. Each operation can be processed by only one machine, in the manufacturing context, and a trip can only be allocated to a vehicle in the transportation area. Machine breakdowns and delay in operations can be approached as vehicle breakdowns and trip delays, respectively. Based on these similarities, the TRP was formulated as a job shop problem with additional constraints (Sahin 1999; D'Ariano et al. 2007a; D'Ariano et al. 2008). The most interesting additional constrain is the so-called blocking constraint. While in the machine context, infinite buffer capacity can be considered between two consecutive machines, this is not the case in transportation. However, the real-time machine recovery problem presents some peculiarities, such as the possibility of changes in the routes of jobs and flexible machines. As a result, this problem is very difficult to solve optimally, given the large number of alternative solutions, even for a very small number of machines and jobs. The real-time machine recovery has been solved using mainly heuristic procedures, based on artificial intelligence techniques, and simulation. As a consequence, the real-time machine recovery methods and techniques are having little impact on road vehicles and aircraft recovery problems, where optimization-based methods prevail.

The complexity of the problem is related to the number of rescheduling alternatives currently assigned trips, viewed as binary variables in a mathematical programming formulation. The difficulty of solving integer programming problems with a large number of variables is well known. The literature presents complexity analysis for some classes or subclasses of the RTVSRP. Li et al. (2009) showed that the VRSP is an NP-hard problem. Corman et al. (2010) demonstrated that the train conflict detection and resolution (CDR), a sub-class of the TRP, is NP-complete, while Berger et al. (2011b) proved that the online railway delay management (ORDM) problem is PSPACE-hard (Arora and Barak 2009). 
Fig. 4 Scheduling and disruption decision-making process (Li et al. 2007a)

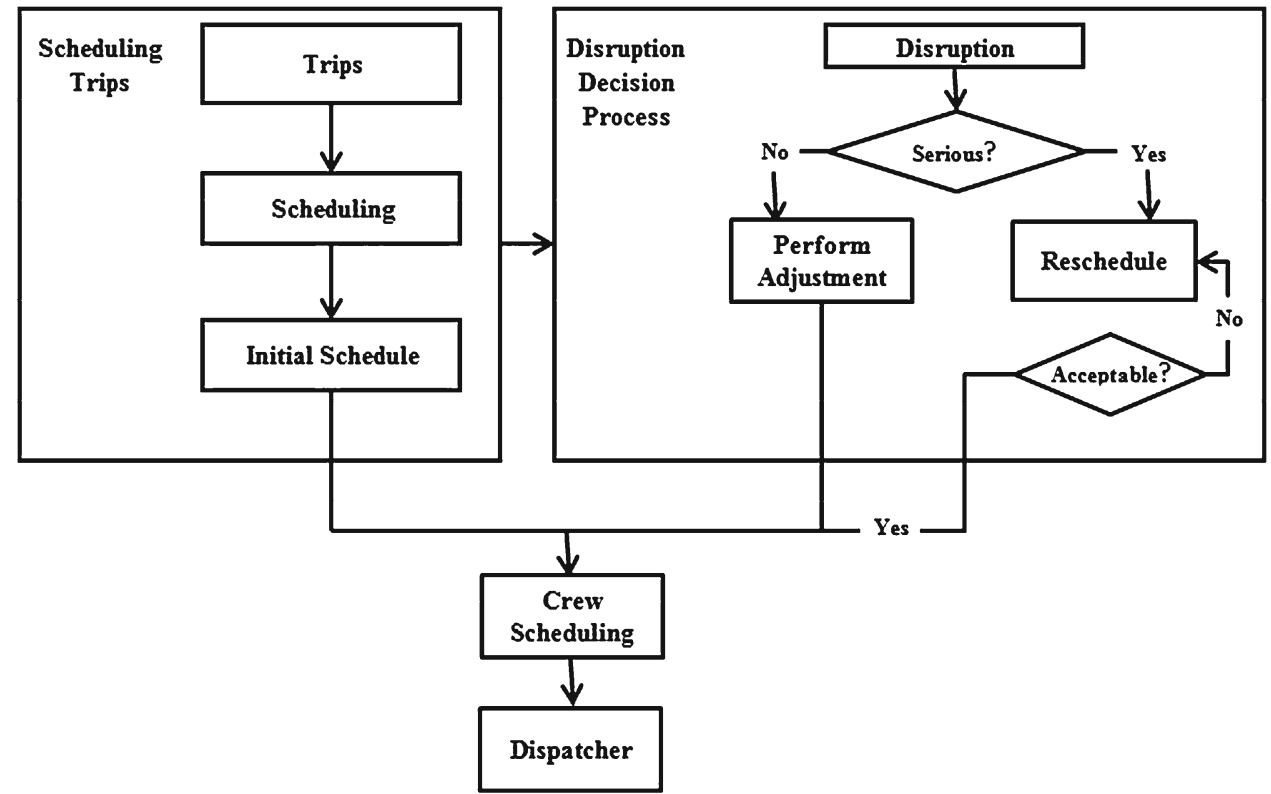

Concerning the aircraft rescheduling problem, Liu et al. (2008) and Babić et al. (2010) showed that the problem is NP-hard for multi-fleet scheduling, while Luo and Yu (1997) showed the NP-hardness of the problem when ground delay is the considered disruption.

The most important aspect of the RTVSRP is the dynamic environment in which the rescheduling process has to take place. That is, while the rescheduling is being performed, the status of the transportation system is changing at the same time. As a consequence, to be used in the real-world, the RTVSRP requires quick solutions. Since the rescheduling problems that have to be solved are large and complex, the realization of short computation times for exact rescheduling algorithms is still a large scientific challenge (Kroon and Huisman 2011). The next sections discuss how these challenges have been addressed in the RTVSRP for ground services, trains, and airlines.

\section{Schedule recovery for road-vehicle services}

In this section, we focus on rescheduling of transportation services that operate vehicles on roads and streets, for example bus services and pick-up and delivery services, when the availability of vehicles decreases due an unforeseen event such as a vehicle breakdown. The VRSP can be approached as a dynamic version of the classical vehicle scheduling problem (VSP) except now assignments are generated dynamically. Real-time rescheduling tools should be very useful in helping decision-makers in disruption situations, especially when fleet size is limited, but, unfortunately the numbers of companies/agencies that use computerized rescheduling algorithms for generating new candidate schedules are few, since most of them reschedule vehicles are based solely on the experience of a human scheduler.

This section is divided into two parts: (i) in the first subsection the vehicle rescheduling context and recovery decision process are discussed, and (ii) in the second, schedule recovery models and solution approaches are discussed.

\subsection{Rescheduling context and decision process}

The VRSP arises in the operation context of a bus/truck transportation system when a previously assigned trip is seriously disrupted. Such problem involves the dynamic rescheduling of the fleet, ensuring that all previously scheduled trips are either completed in a reasonable amount of time or canceled. Traffic accidents, medical emergencies, and vehicle breakdowns are examples of possible disruptions that require vehicle rescheduling. Instances of VRSP happen in applications such as school bus transportation, public transit services, industrial/hospital refuse collection, mail delivery, etc.

$\mathrm{Li}$ et al. (2007b) presented a decision diagram showing the interactions between schedule planning, disruption decision-making processes, and rescheduling (Fig. 4). First, the scheduling process can be characterized as the classical single-depot VSP (SDVSP) when only one depot is involved (otherwise it is the multi-depot version). Second, the severity of disruption is defined by the experience of human schedulers. Small disruptions usually need few adjustments and, in general, the initial schedule is not changed by much. Otherwise, when the disruptions are larger, this initial schedule is used as a basis for rescheduling the remaining unfinished and future trips; then the current schedule could change substantially. We notice that, in order to develop a rescheduling decision support, information technology is essential to capture 
information on disruptions, such as time and place disruption, number of passengers or vehicle cargo size, and subsequently to quickly compute and communicate new candidate schedules. Much of this schedule recovery process applies also to the other two domains reviewed in the paper-rescheduling of trains and planes.

\subsection{Vehicle rescheduling models}

Bunte and Kliewer (2009) presented a comprehensive overview on vehicle scheduling models and found that much research has been done on it in the last decades, but considerations on vehicle rescheduling are still relatively unexplored. Major contributions toward solving VRSP are found in Huisman et al. (2004) and Li et al. (2004).

Huisman et al. (2004) considered an environment with significant traffic jams and developed a cluster-reschedule heuristic to solve what they called a "dynamic" VSP problem. The "dynamic" aspect was mainly related to delays in the previously assigned trips. The problem formulation was based on the multi-depot VSP (MDVSP) and solved as a sequence of optimization problems. First, trips were clustered using the static VSP, and then rescheduled for each depot. Real data of a public transport company was used to test their approach. The results indicated that the number of trips starting late was reduced at the price of using a few extra vehicles. In a later paper, Huisman and Wagelmans (2006) integrated the dynamic VSP with crew scheduling, solving the integrated system dynamically. Two algorithms were developed. The first one uses a sequential approach (first schedule vehicles and then schedule crews); the other uses what the author called an "integrated approach." The integrated approach consists of solving a sequence of integrated vehicle and crew scheduling problems, formulated as binary linear programs. Historical data from a large bus company in the Netherlands for a period of 10 days were used for some computational tests. Two problem sizes were tested: a small single-depot (164 trips) instance and a medium-sized multiple-depot (304 trips) instance. The integrated approach significantly outperformed the sequential approach for the larger problems; for the smaller problems, the performances were not that different. A limitation of the integrated approach is that computation times may be too high for practical real-time operations.

In Huisman's papers, delays on trips due to disruptions were the major considerations in their predictive rescheduling strategies. A different approach is taken by $\mathrm{Li}$ et al. (2004), which considers disruptions due to vehicle breakdowns or severe vehicle accidents for schedule recovery, introducing the reactive strategy for this problem class. The problem is modeled as several VSPs, each one corresponding to the use of a different vehicle as an alternative for backing up the disrupted trip. Each backup vehicle $k \in K$ generates a $\mathrm{CN}, G(k)$, referred as a feasible network. The paper describes an algorithm to find all possible backup vehicles for a serious disruption, considering the peculiarities of the transportation service being addressed, for example, bus routes may partly share the same itinerary. In order to solve the problem, a parallel auction algorithm has been developed. The parallel algorithm has proven to be computationally efficient in random generated instances up to 1,300 trips. Li et al. (2007a) improved the solution time of the parallel auction algorithm by introducing the common feasible network (CFN) notion. The CFN can be obtained from the intersection of all possible feasible networks as follows: $C F N=\cap_{k \in K} G(k)$. The idea is to find a good set of initial "prices" using the CFN, characterizing a better initial assignment, to speed up the auction algorithm. The algorithm performs well for a large number of trips and vehicles. However, the modeling approach was based on the following two restrictive assumptions: (i) rescheduled trips, except the disrupted trip, cannot suffer delays; and (ii) there are no restrictions on the number of trips that may be reassigned. In practice, these assumptions reduce the size of the problem, restricting the number of possible rescheduling alternatives.

In order to minimize the restrictive assumptions involving auction-based algorithm, Li et al. (2009) developed a Lagrangian heuristic approach for the VRSP. The heuristic approach incorporates Lagrangian relaxation, a sub-gradient search and an insertion-based primal heuristic. The developed formulation expands the applicability of the model by simultaneously taking into account the previously neglected real-world aspects such as reducing trip cancelation costs, and decreasing the number of rescheduled trips (toward facilitating the crew rescheduling, since several bus crews will be familiar with the complete itinerary of a subset of all possible trips). Li et al. (2009) observed that the Lagrangian heuristic did not demonstrate many improvements in situations where a large number of remaining trips exist, when a backup vehicle is available at the depot, or when the vehicle breaks down at the beginning of the disrupted trip.

The importance of rescheduling in practice was also recognized by Li et al. (2007b, 2008). In Li et al. (2007b) a decision support system (DSS) was developed to facilitate a practical application for rescheduling trucks for solid waste collection in a Brazilian city. The approach helps to solve the complex problem of recovery from severely disrupted trips, minimizing the involved disruption costs. The SDVRSP was treated as a sequence of SDVSPs. A quasiassignment formulation and a combined forward-backward auction algorithm were used to model and solve, respectively, the SDVSPs within the DSS. CPLEX was used to solve computational experiments with randomly generated data. The results indicated that the DSS has potential as an effective and efficient tool for real-time operational planning in transportation/logistic companies. In a following paper, $\mathrm{Li}$ et al. (2008) proposes a nonlinear programming formula- 
tion for the waste-collection problem, minimizing not only disruption costs, but also trip delays. The big- $M$ technique was used to linearize the formulation, simplifying its solution. The main objective of the model was to minimize the sum of operation costs, fixed vehicle costs and delay costs, under the conditions that all incomplete trips have to be finished (including the disrupted one), and trip assignments into waste-recycling facilities were balanced. In that paper, each vehicle operating in the network at the time of the disruption could be considered as a pseudo-depot with unit capacity, making the mathematical formulation now being based on the MDVSP. Experiments based on real-world data were carried out using CPLEX as the commercial solver, since the instances used were small, consisting of 23 vehicles and 31 trips. The developed model decreased both the total distance traveled and total trip delay when comparing with the manual recovery strategy used by the company.

Ernst et al. (2007) developed a software to solve the dynamic scheduling of vehicles, referred to as the Dynamic Vehicle Assignment and Scheduling System (D-VASS), assisting a large New Zealander recreational-vehicle-rental company in its daily operations. The main purpose of D-VASS was to respond to availability queries by reservation staff and to incorporate new bookings into the schedule. It also adjusted the schedule in response to operational contingencies, like vehicle breakdowns and late returns. D-VASS applies a heuristic improvement method based on the successive shortest path for solving the assignment problem and perform updates as quickly as possible. The model only considered the beginning and end data of a trip, but did not consider important information like the place and time that the vehicle suffers the disruption; also public transport applications were not considered.

Table 2 summarizes the reviewed literature and highlights some attributes of interest in the developed models. In sum mary, the models, formulations, and solution procedures developed for the VRSPs are much related to their static counterpart, mostly using the connection network as the representation of the problem, and the MDVSP solution methods as inspiration for the design of algorithms for the real problems. The applications of VRSP include the scheduling of buses, trucks, and rental vehicles (Ernst et al. 2007). The computational experiences reported include some large problem instances, up to 1,300 unfinished trips when a disruption occurs.

\section{Rescheduling train services during disruptions}

Rail transportation provides services for passenger traffic and freight shipping. However, train scheduling is a difficult problem primarily due to its size and the significant interdependencies between the train movements and operational constraints.

\subsection{Train rescheduling considerations}

Jespersen-Groth et al. (2009) noted that trains do not always run on time due to unexpected events like infrastructure malfunctions, rolling stock break downs, accidents, and weather conditions. The so-called "snow ball effect" (that is a deviation from the planned operations of a train which can have a cascading effect on several other trains' operations in the surrounding traffic area due to sharing of physical resources, such as tracks and stations) is a major complexity in train rescheduling. The compounding factors include (Acuna-Agost et al. 2011; Tornquist and Persson 2007):

a. Trains cannot share the same track in opposite directions at the same time. To avoid collisions, railway networks are often composed of blocks, which are track sections that can be used by at most one train at a time due to safety restrictions. The lengths of block segments are variable and are dependent on traffic density. To increase safety, a buffer space of one or two blocks between two trains is exerted by several train control systems;

b. There is a much diversity in railway infrastructure; some blocks are single-tracked, others are double-tracked or even $n$-tracked;

c. The train traffic is quite heterogeneous; heavy cargo trains share tracks with passenger trains;

d. There are minimum and maximum idle times of trains at each station. Although these values can be changed in case of a disruption, it can cause itinerary problems to passengers.

For more details on the constraints related to train scheduling refer to Cordeau et al. (1998) and Jespersen-Groth et al. (2009).

The TRP consists in defining a new re-allocation of trains to blocks, modifying the original timetable while respecting all the required technical and commercial constraints and (usually) attempting to minimize the total train (or passenger) delay. In addition, given the real-time aspect of the problem, the new schedule has to be consistent with the current state of the system and has to be generated very quickly, since the solution must take into consideration that some trains are already delayed at the moment, the new schedule is implemented (Acuna-Agost et al. 2011).

Jespersen-Groth et al. (2009) introduced a schematic view of the train disruption management process (see Fig. 5). The process can be briefly summarized as follows: when a disruption occurs, the Network Traffic Control (NTC) needs to decide about the final dispatching plan for all trains in the network and communicate it to the Local Traffic Control (LTC) and to the operators. The LTC has to implement the new train routes and to change platform assignments, accordingly. 


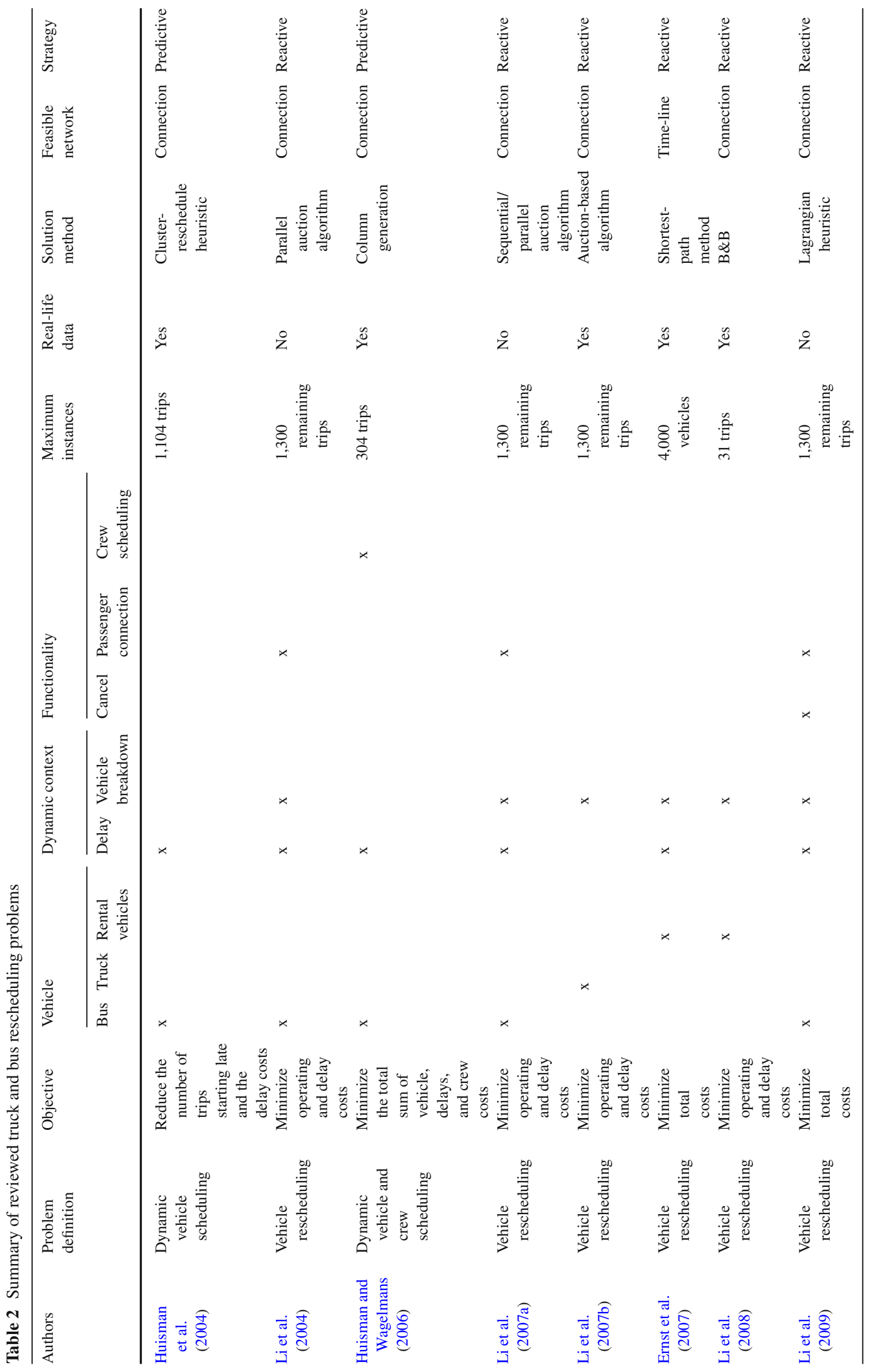


Fig. 5 Train disruption management (Jespersen-Groth et al. 2009)

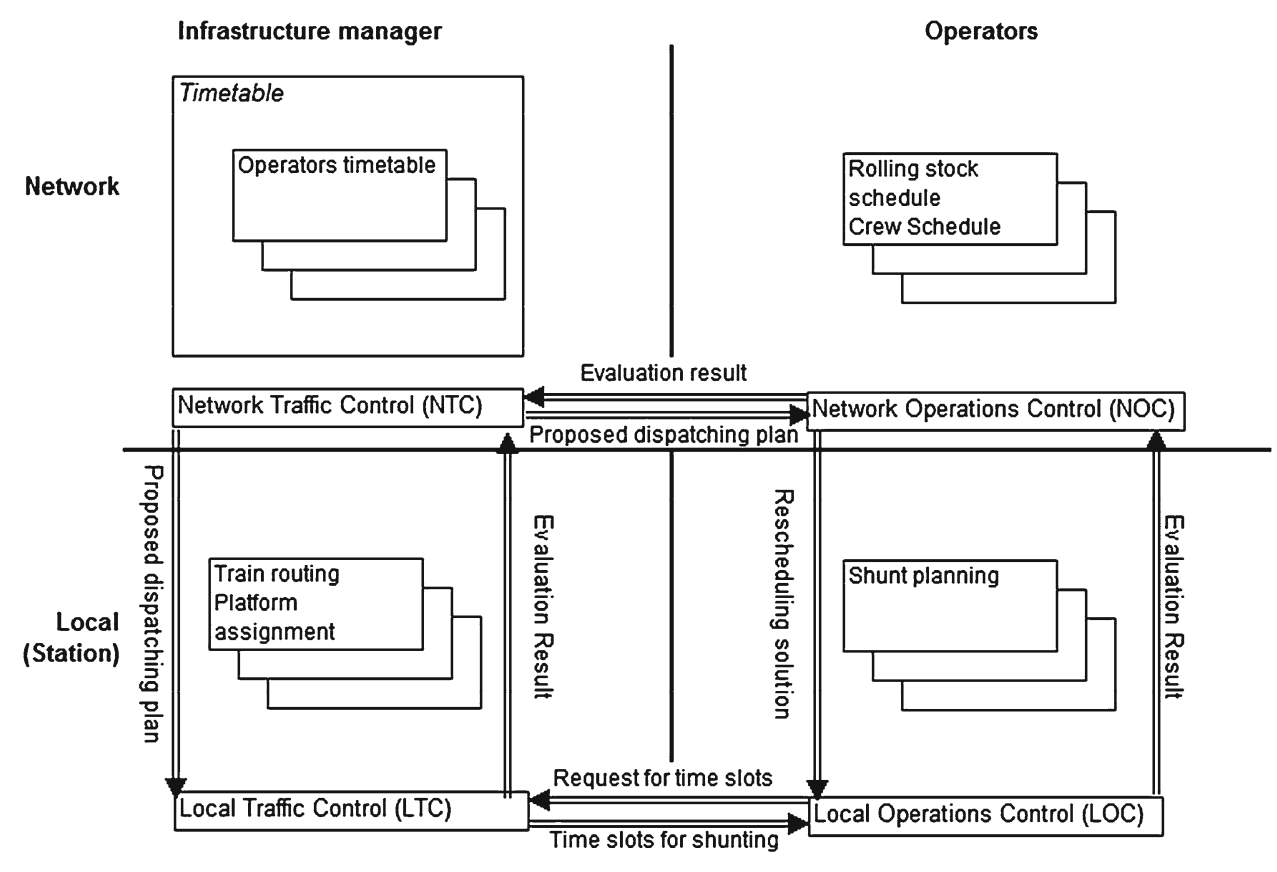

The Network Operations Control (NOC) monitors and modifies the rolling stock and crew scheduling based on the new train routes. The Local Operations Level coordinates the local activities at the stations, such as the shunting processes.

Until recently, the TRP was solved manually, evaluating a limited number of possible corrective actions of reordering and/or rerouting trains. The increased capacities and speeds of computational resources available and the recent advances on solving large integer linear programming problems have resulted in algorithmic-based decision support that quickly gives better solutions. The next section will briefly discuss some of these TRP solution methods. Since railway crew rescheduling problem is beyond the scope of this review paper, we refer to Potthoff et al. (2010) and Veelenturf et al. (2012) for this related topic.

\subsection{Train rescheduling models}

Törnquist (2006) has presented an overview of research on train scheduling and dispatching. Of the reviewed 48 articles, published between 1973 and 2005, 21 dealt with rescheduling. The major objective of the reviewed models was the minimization of the total delay. Some small variations, like the introduction of weights to emphasize the delay of some trains, are also addressed. The reader is referred to Törnquist (2006) for a pre-2005 review of train rescheduling; this review focuses on train rescheduling work reported after 2005 .

Different infrastructure representations have been addressed, including lines and general networks, bidirectional or unidirectional tracks, parallel tracks, etc. In general, the most complex models cannot deal efficiently with more than 30 trains. Table 3 presents a summary of the main characteristics of the reviewed papers. Unlike the VRSP, which usually relies on mathematical programming solution approaches, TRP methods reported include a greater variety of approaches. Improvement heuristics, construction heuristics, and simulation have been used as alternative methods to find good solutions in reasonable time, which are justified due to the complexity of the real-world TRPs. An interesting aspect of contemporary literature on the TRP is that almost all papers present experimental evaluations with real-data, demonstrating the applicability of the developed tools.

Tornquist and Persson (2007) developed an interesting mathematical formulation for the rescheduling of $n$-tracked railway traffic. The railway network studied included several merging and crossing points. The problem was formulated as a mixed-integer linear program (MILP), based on an eventdriven representation of time, and solved using CPLEX 8.0. An event was defined as the conditional resource request by a train for a segment of a track. Two alternative objective functions have been considered: minimize the total final delay of traffic, and minimize the total costs associated with delays of trains at the final destinations. Since the authors noticed that only few modifications in the initial timetable were necessary to obtain a good solution, different strategies, such as "allow swaps of tracks but maintain order," were evaluated on large practical size problems. The solution approaches, based on different strategies, seemed to perform well with respect to run-time efficiency and solution quality in several cases. Acuna-Agost et al. (2011) proposed an extension of this model, allowing the possibility of two trains in the same direction to use the same track segment simultaneously. The MILP formulation presented, which used the same notation as Tornquist and Persson (2007), attempts to 


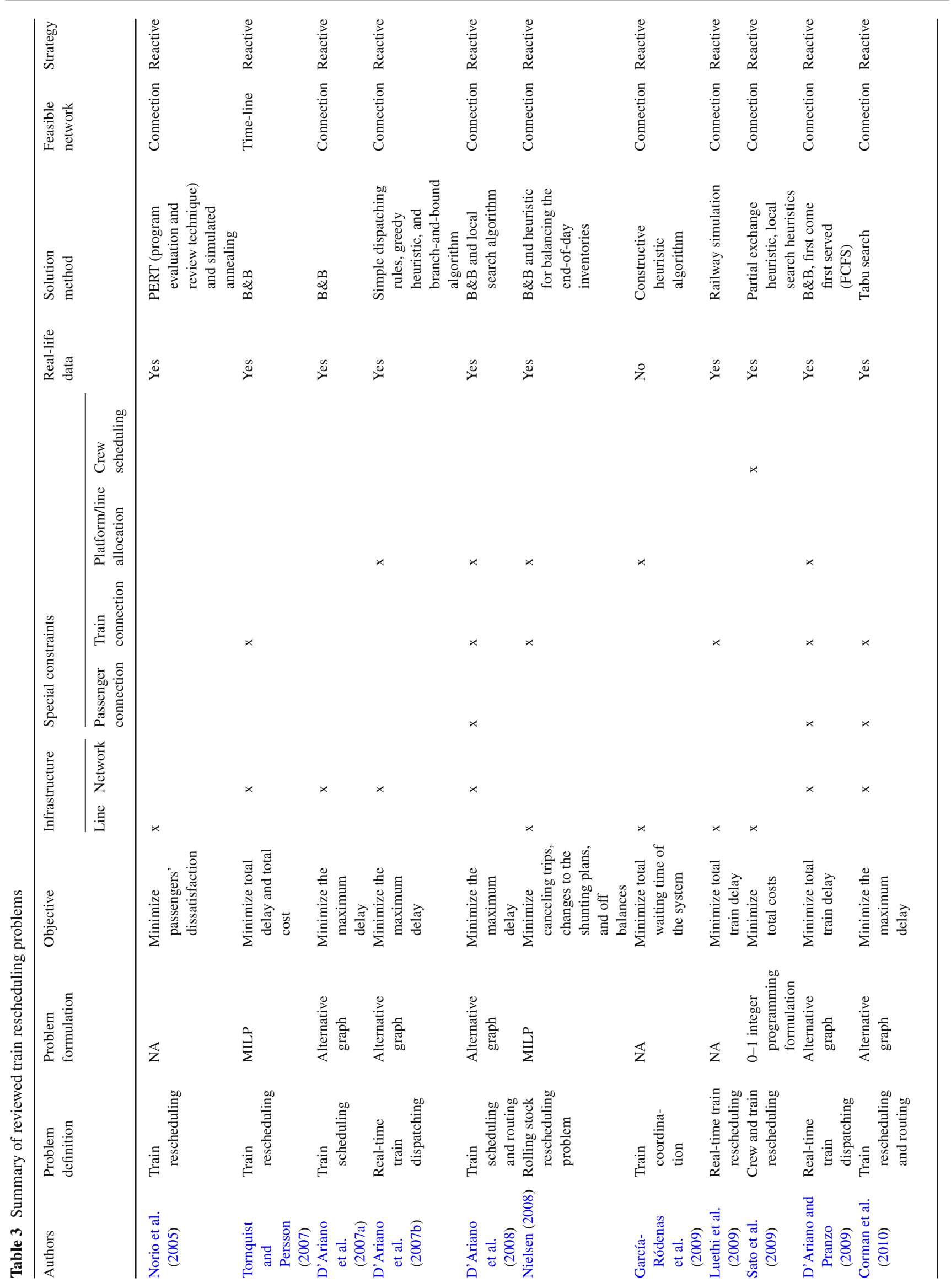




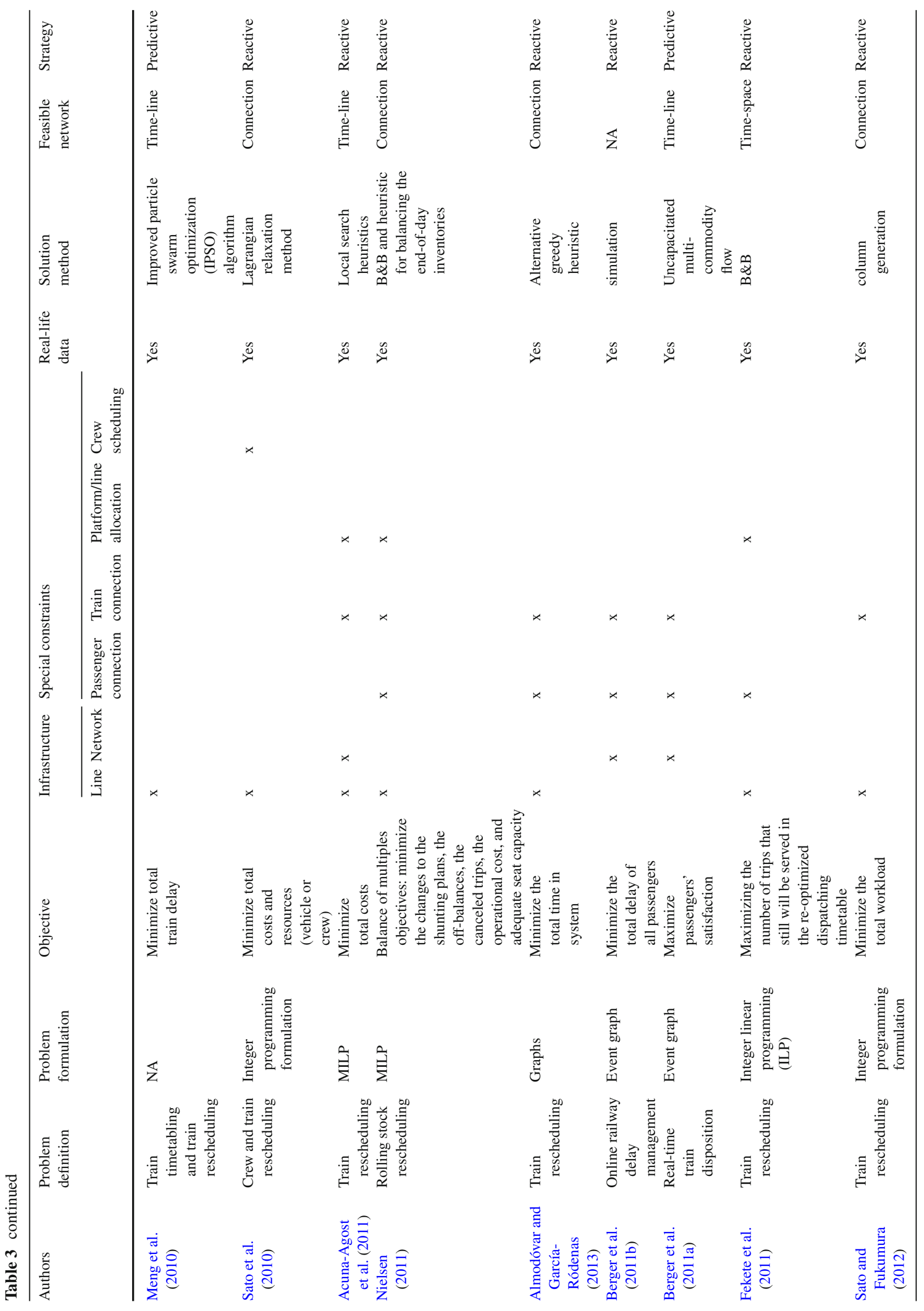


minimize the total rescheduling cost based on the total delay. The performance of four solution methods were evaluated and compared as follows: (i) Right-shift rescheduling (RS) keeps most of the system characteristics, postponing each remaining trip after disruption by the amount of time needed to make the schedule feasible, limiting the propagation delays; (ii) MILP-based local search method using RS as initial solution; (iii) IP-based local search method + CPLEX; and (iv) Iterative MILP-based local search method (LS). Two different real rail networks have been used for the computational experiments: the first is a line located in France, and the second is a railway network in Chile. The Iterative MILPbased local search procedure was able to obtain better solutions (average gap $<1 \%$ ) within 5 min of computational time; thus it seems to be viable for real-world situations.

Nielsen (2008) presented a generic framework for the rolling stock rescheduling problem, when several changes to the timetable may occur and the circulation has to be updated every time. The problem is formulated as a MILP. The objective function minimizes simultaneously the number of canceled trips, changes to the original rolling stock plan, and changes on the planned end-of-day balance of rolling stock on each depot. The instances used in the experiments come from rolling stock circulations used by Netherlands Railways and solved using CPLEX 10.1. The observed computation times varies from a few seconds to a minute depending on the size of the considered instance. There is a direct positive correspondence between the experienced computation times and the length of the considered time horizon (in hours). In his $\mathrm{PhD}$ thesis, Nielsen (2011) studied the disruption management in a context of rescheduling of passenger railway rolling stock. The model contemplates real-life aspects and penalizes cancelations of trains, changes on shunting processes, carriage kilometers, seat shortage kilometers, as well as deviations from the planned end-of-day rolling stock off-balances on each depot. The previously developed model is expanded considering the dynamics of passenger flows and incorporates the passenger behavior by applying an iterative solution procedure that unifies the optimization of the rolling stock assignment with detailed simulation of the passenger flow. All the tested instances were based on real data from one of the Dutch railway operators.

Berger et al. (2011a) developed an optimization tool for solving the train disposition problem, concerned with defining whether a train should wait for an incoming delay train or not. The problem is represented using an event-graph and formulated as a variant of the uncapacitated multi-commodity flow. The major objective of the model is the satisfaction of passengers, through three different objective functions as follows: (i) the overall lateness at the destination, (ii) the deviation from original travel plans of passengers, and (iii) the number of passengers that do not reach their destination. The authors carried out experiments, considering real and artificial delay scenarios (based on data from German Railways). The results have demonstrated that the approach is fast enough to be applied in a real system, enabling quick decisions.

Fekete et al. (2011) employed an ILP programming formulation based on the event-activity network, suggested by Serafini and Ukovich (1989), for schedule recovery of railbased urban mass transit systems. It focuses on scenarios where the system faces a bottleneck, such as the direction of a track is shutdown. The formulation incorporates constraints to cope with unplanned turns or unplanned returns to a depot. The rescheduling objective was to maximize the number of trips that will still be served after the disruption. Since frequency of most subway systems is high and canceling few trips lead to only minor delays and inconveniences for the passengers, minimizing the overall delay was only a secondary objective. Four scenarios of real-life instances have been evaluated, differing in shutdown location, transit times, topology of the switches, and turn possibilities. CPLEX was used to solve the problems. This model has the potential for real applications since good quality solutions were found within a minute.

Some ad-hoc heuristics have been suggested for solving the TPR. Norio et al. (2005) proposed a heuristic algorithm for automatic train rescheduling, in which the main objective is to minimize passengers' dissatisfaction measured by a planned dissatisfaction index. The algorithm combined simulated annealing (SA) and Program Evaluation and Review Technique (PERT). The PERT module enabled reducing the search space for the SA procedure, resulting in an efficient algorithm. To test the algorithm, a whole-day schedule for 564 trains was developed for an urban line in Tokyo, about $40 \mathrm{~km}$ long and containing 19 stations. New schedules were obtained in approximately $1 \mathrm{~min}$ for recorded disruptions. Meng et al. (2010) used particle swarm/genetic algorithms for train timetabling and train rescheduling. Three different algorithms were developed: normal particle swarm optimization (PSO) algorithm, genetic algorithm, and improved particle swarm optimization (IPSO). The algorithms were evaluated in double lines autolocking section in China, considering five stations and 60 trains. The best results were obtained by the IPSO algorithm.

Almodóvar and García-Ródenas (2013) studied a train capacity problem where a service line is substantially disrupted due to a heavy unexpected demand that exceeds the service line capacity. A greedy heuristic and an event-based simulation model were applied to determine the best vehicle reassignment decisions. This approach provides nearoptimal solutions, but it involves a high computational cost and does not currently seem applicable for real-time response. García-Ródenas et al. (2009) also used simulation models for schedule recovery. The authors dealt with on-line management of public transport systems under disruptions, 
such as non-recurrent congestion and in situations in which demand is greater than the capacity offered by the current transit line. Their problem was to decide which vehicles, and in which time instants, must be reassigned to the disrupted line in order to minimize the waiting time in the system. Some train network constraints were not taken into consideration to decrease the complexity of the problem. Their online optimization approach was based on a predictive simulation model, a queuing model, and a constructive heuristic algorithm. Luethi et al. (2009) developed a simulation model for the real-time train rescheduling aiming at reducing buffer times without impacting schedule reliability. The simulation was carried out on a critical bottleneck block in the Swiss rail network. Simulation experiments show that a real-time rescheduling system could significantly reduce the total delays even in the case where additional trains are added to the timetable. Simulation has also been used by Berger et al. (2011b) in order to solve the ORDM problem, in which passengers have a fixed network route, but are allowed to adapt the choices of the trains based on delays. The major objective is to minimize the total delay of passengers. A websimulation platform has been developed where a simulation framework and a heuristic approach have been integrated to evaluate and compare different heuristics for solving the ORDM considering stochastic delays. The model design and application were directed to a robust rather than a real-time context, developing a powerful tool to design policies for coping with railway delay management.

Sato et al. (2009) added the crew rescheduling problem to the TRP. They set up a scenario in which a train broke down between stations for $2 \mathrm{~h}$, resulting in a large-scale disruption and several timetable changes, such as canceled train services and extra trains. The problem was formulated as an integer programming problem. The solution was based in a twophase approach. The first one uses a partial exchange heuristic, generating a feasible solution by modifying the original schedule. The second approach uses a local search for alternative solutions to search for alternatives that improve an evaluation value that includes crew scheduling effects, while maintaining the schedule feasibility. The results of numerical experiments with real-world data showed that the proposed method generated feasible solutions within a practical amount of time, and the two-phase solution approach improved the solution quality. Later, Sato et al. (2010) used a Lagrangian relaxation as a solution mechanism for the crew and VRSP, formulated as network flow models. They used real-world data from a Japanese railway line to conduct numerical experiments for vehicle rescheduling.

Recently, Sato and Fukumura (2012) focus on the rescheduling of locomotives that haul freight trains in Japan. Two problems have been formulated as integer programming models, namely the train rescheduling problem and the uncovered train detection problem. Both problems were solved by column generation. They tested the model performance with real data obtained in a Japan railway line with high frequency. The results show that the proposed algorithms provide satisfactory solutions within $30 \mathrm{~s}$ on a PC (32-bit Windows PC Core i7 CPU, 3.2 GHz, 3 GB RAM) for several studied cases.

Several papers in the TRP literature are specially focused on the CDR problem in network railways, which is related to the real-time train scheduling and routing (D'Ariano et al. 2007a,b; D'Ariano et al. 2008; D'Ariano and Pranzo 2009; Corman et al. 2010). The CDR consists of changing dwell times, train speeds as well as train ordering and routing (Corman et al. 2010). Their modeling and solution methods use alternative graph formulations (Mascis and Pacciarelli 2002). Alternative graph was especially developed to cope with the scheduling problems where the response time is a critical factor for the evaluation of a method. An example, based on a slightly larger one presented in Mascis and Pacciarelli (2000), illustrates the alternative graph. Figure 6 shows a small railway with two trains traveling in opposite directions. Train A is moving from block section 1 to 10 , while train B is moving from section 9 to 1 . Train B needs to pass through platform 4 . This railway has four block sections (1, 7,9 , and 10), three junctions $(2,5$, and 8$)$ and trains $A$ and B share four resources (sections 2, 5, 6, and 8).

Figure 7 shows the alternative graph for this small railway. For sake of clarity, each node of the alternative graph is indicated with a pair (train-block section), e.g., A2 indicates that train $\mathrm{A}$ is traversing block section 2 . There are four pairs of alternative arcs, represented by connecting the two paired arcs with a small circle. The initial position of train A implies that train B is not allowed to precede train A in section 2 . The required time to pass through all track segments is represented by $\alpha$.

The main advantage of the alternative graph formulation is the detailed representation of the network topology at the level of railway signals and operational rules. D' Ariano et al. (2007a) propose a truncated branch-and-bound (B\&B) algorithm for the CDR problem with fixed routing, aiming to minimize the maximum secondary delay for all trains at all visited stations. Due to the interaction between trains, delays caused by technical failures and disturbances may be propagated to other trains in the network, defined as secondary delays.

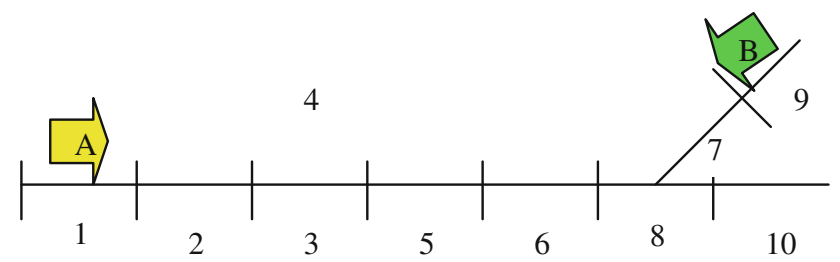

Fig. 6 A small railway network for two trains in the opposite direction (based on D'Ariano and Pranzo 2009) 
Fig. 7 The alternative graph for the sample railway (based on D'Ariano and Pranzo 2009) $-\boldsymbol{\alpha}$

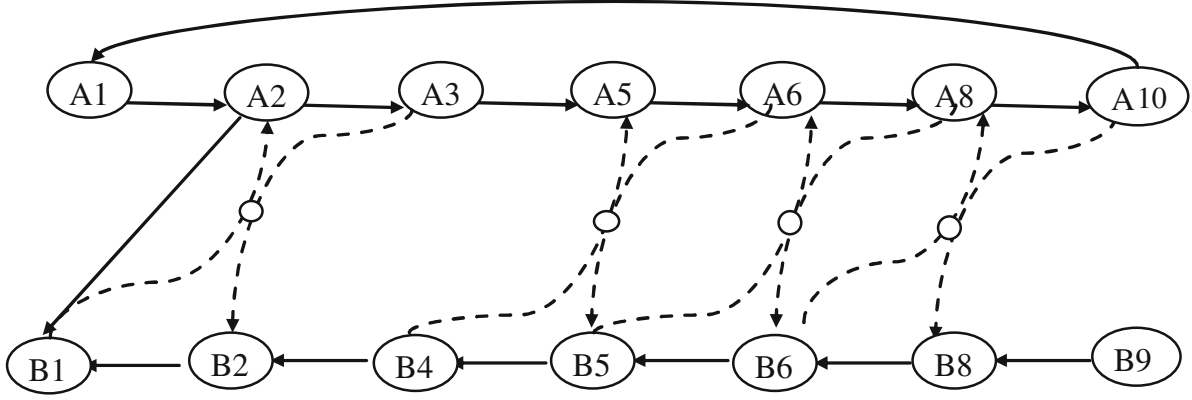

Computational experiments were carried for a real rail network and for multiple delayed trains. The model incorporates a detailed description of the network topology, including railway signals and safety rules. Effective static implementation rules for conflict resolution problem were developed. It resulted in a significant reduction in the computation times obtaining near-optimal schedules for practical size problems. D'Ariano et al. (2007b) also studied a variable speed model that coordinated speed among consecutive trains, based on the alternative graph formulation where safe distance headway between trains is respected. The model simultaneously considers speeds of all trains with the objective of minimizing the maximum delay due to conflicts. Three approaches were used to solve the problem: simple dispatching rules, a greedy heuristic based on the alternative graph formulation, and $\mathrm{a} B \& \mathrm{~B}$ algorithm. B\&B provides better quality solutions, but requires more computation time. Moreover, the results indicated that fixed-speed model underestimates the consequences of braking and acceleration, while the variable-speed model presents more realistic solutions.

In D'Ariano et al. (2008), D'Ariano and Pranzo (2009), and Corman et al. (2010), rolling stock and passenger connections are included in the models. D'Ariano et al. (2008) described the traffic management system railway traffic optimization by means of alternative graphs (ROMA) which is able to solve the CDR problem in real-time for moderate size dispatching areas. A B\&B algorithm was used to sequence train movements, while a local search algorithm was used for rerouting optimization purposes. Computational tests, carried out for a Dutch dispatching area between Utrecht and Den Bosch, including instances with multiple delayed trains and different blocked tracks in the network, showed that significant delay reductions were achieved by the rescheduling of train movements.

D'Ariano and Pranzo (2009) extended ROMA to proactively evaluate the effects of train rescheduling actions for short-term prediction of train traffic. The truncated B\&B algorithm introduced by D'Ariano et al. (2007a) was reapplied with the first come first served scheduling rule by D'Ariano and Pranzo (2009). The authors focused on disturbances caused by train delays and temporary unavailability of some tracks. Since the prediction of railway traffic can result in computationally intractable instances, the time horizon was decomposed into smaller time intervals that are solved sequentially, with the objective of improving train punctuality. To detect and solve conflicts at each time interval, the ROMA dispatching system was employed. The independent solution of each hour of dispatching permits handling large time horizons with a linear increase of computation time. This approach was compared with a formulation in the problem for the full-time horizon, in order to evaluate the error due to the problem decomposition. The temporal decomposition proved to solve large scheduling problems; however, the full time-horizon solution gave smaller time delays but with much larger solution times.

Corman et al. (2010) considered passenger connections, multiple delayed trains, and heavy network disruptions. With the objectives of increasing the solution quality and reducing computation times, in comparison of the approaches in D'Ariano et al. (2007a) and D'Ariano et al. (2008), they developed a tabu search strategy. Their experiments on practical size problem instances, such as the Dutch dispatching area between Utrecht and Den Bosch, showed that (i) the tabu search strategy decreased the optimality gap for small instances where the optimal solutions were known, and (ii) for large instances, the solution quality was better and computational times were lower.

\section{Airline schedule recovery problem}

The airline recovery problem is the most studied class of problems in the RTVSRP. Since a disruption in the airline traffic could have severe operational and economical consequences (Ball et al. 2007), the development of fast and reliable recovery methods is of much interest to airline companies. In particular, airline passenger companies have become very interested in this problem since passenger delay is a major issue as the growth in air transportation has outpaced the capacity at some busy airports (Petersen et al. (2012)). The development of optimization models for airline schedule recovery has been a challenge to the O.R community since 
Fig. 8 Airline disruption management (Liu et al. 2008)

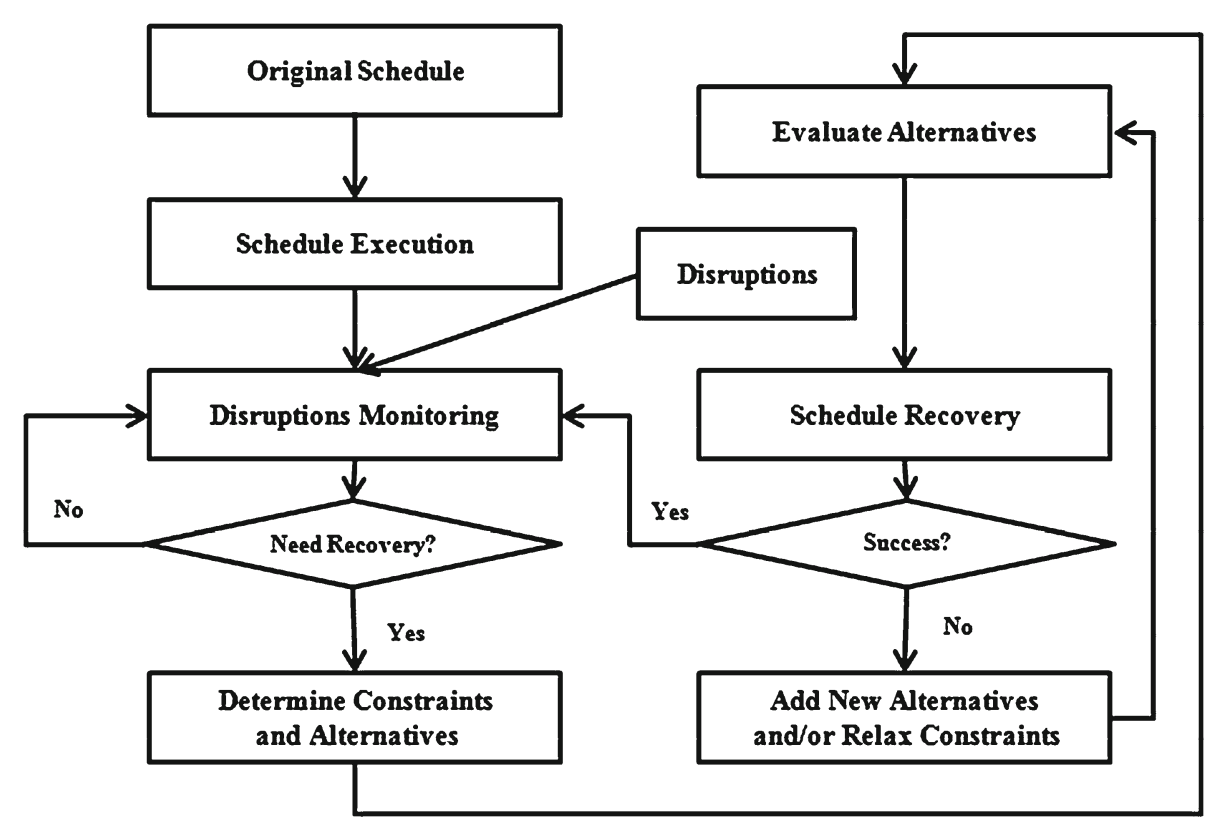

the eighties; Teodorovic and Guberinic (1984) is perhaps the most cited early work. Since then, several optimization and heuristic rescheduling methods have been developed.

\subsection{Aircraft rescheduling considerations}

The process of airline disruption management, developed by Liu et al. (2008) is shown in Fig. 8. A small disruption might require no rescheduling, due to the inherent flexibilities and slack in the original schedule. However, if the deviation between the disrupted and original schedule becomes large, rescheduling becomes a necessity. Unplanned events that impact airport infrastructure and air traffic control, extreme weather conditions, and emergencies to maintain safety are examples of disruptions that can ripple throughout the system, resulting in cancelation or delays. Three types of disruptions - that could occur concurrently - might disturb a planned aircraft schedule (Bisaillon et al. 2011):

1. Flight disruption — when a flight is delayed or canceled.

2. Aircraft disruption - when an aircraft is unavailable for a period of time.

3. Airport disruption - the departure or arrival capacities of an airport are temporarily reduced.

Often when an airport disruption occurs, a Ground Delay Program (GDP) is issued at this airport. The GDP purpose is to increase the time gap between successive flight landings in order to ensure safe operations during the adverse conditions period. Under most GDPs, the available number of slots for flight landings becomes less than planned. Therefore, a scheduled flight could be held at its origin, diverted to a nearby airport, or in the worst case it could be canceled (Abdelghany et al. 2008).

When a severe disruption occurs, many resources (crew, aircraft, passengers, slots, catering, cargo, etc.) have to be rescheduled. Large airlines usually react to this by solving the rescheduling problems in a sequential fashion. First, the aircraft rescheduling problem is solved. Next, the crew pairing problem is solved. Finally, a solution for the crew rostering problem is defined. Although the three rescheduling problems are highly related, this paper focuses on the ARP. We refer to Clausen et al. (2010) for an extensive review of disruption management in the airline industry.

An ARP solution gives a new route for each aircraft, given that a serious disruption occurred. The new routes must comply with several technical, commercial, and regulatory constraints based on rules defined by national and international aviation organizations. Airline companies' policies, aircraft capacity, and airports' conditions typically define constraints to be taken into consideration when determining a revised schedule. The solution of the problem gives a set of feasible routes that can minimize the adverse effects of the disruption.

\subsection{Aircraft rescheduling models}

As mentioned earlier, Clausen et al. (2010) provide an excellent survey on airline disruption management. In their literature review of ARP, 34 papers identified were published between 1984 and 2008. The authors concluded that the majority of the mathematical models and solution methods for solving the ARP are similar to the methods applied for schedule planning. The ARP was formulated based on network flow models (Jarrah et al. 1993; Cao and Kanafani 
1997a,b), TLN (Yan and Yang 1996; Thengvall et al. 2000), TBN (Argüello et al. 1997; Bard et al. 2001), and the set partitioning problem on CNs (Rosenberger et al. 2003; Andersson and Värbrand 2004). To solve the formulated models, solutions methods based on the out-of-kilter algorithm (Mathaisel 1996), B\&B (Bard et al. 2001), Lagrangian relaxation (Yan and Yang 1996; Andersson and Värbrand 2004), greedy randomized adaptive search procedure (Argüello et al. 1997), and tabu search (Andersson 2006) have been proposed. Most models and methods have been evaluated using real-life problem instances. Although most of the algorithmic approaches are capable of considering several important real-life requirements, the solution times of many of them were not practical for real-time applications.

For the sake of economy and to restraint excessive overlaps with previous reviews, we focused on papers published from 2000 onwards. Bard et al. (2001) and Clausen et al. (2010) offer comprehensive reviews of earlier paper related to ARP. Table 4 summarizes the reviewed papers. With the exception of Ionescu et al. (2010), analyzed on section 2, all reviewed papers applied a reactive strategy.

Since weather-related disturbances are the main causes of disruptions in the airline industry (Rosenberger et al. 2003), aircraft disruption has been receiving less attention in the literature compared with flight and airport disruptions. Aircraft mechanical problems are included as possible events causing flight disruptions. Eggenberg et al. (2010) also considered disruptions caused by maintenance events. A column generation algorithm was implemented to solve the problem. The advantage of the developed technique is that it is flexible enough to be applied for aircraft, crew, or passenger recovery problems. The algorithm is based on recovery networks, encoding each unit's (aircrafts, crew, or passengers) feasible route. The computational results show that the algorithm is efficient, solving instances with real data and reasonable complexity in low computation times.

\subsubsection{Flight disruption}

Bertsimas and Patterson (2000) set up a multi-aircraft optimization model minimizing the weather delay cost, based on deterministic weather scenarios. The problem was modeled as a dynamic network flow model with additional constraints. The authors presented a mathematical programming approach referred to as the Lagrangian generation algorithm (LGA). The LGA comprises a Lagrangian relaxation to generate aggregate flows; a randomized rounding heuristic that decompose the aggregate flows into a collection of flight paths for individual aircrafts as well as an integer programming formulation of the packing problem whose solution generates feasible and near-optimal routes for individual flights. The model did not explicitly allow flights to be canceled. Three scenarios were used to evaluate the algorithm efficiency, with problem solution times varying between 116 and $330 \mathrm{~s}$.

Thengvall et al. (2000) expanded the model developed by Yan and Yang (1996), developing an integer network flow model based on TLN to deal with flight delays and cancelations in daily operations. A rounding heuristic was developed to improve the LP-relaxation of the integer formulation. Real data from Continental Airlines for B757 and B737 schedules, solved separately, were used to evaluate the developed model. Good quality solutions were obtained within reasonable computational times, validating its application to realworld cases.

Bard et al. (2001) developed a time-band optimization model for generating new aircraft routings when groundings and delays occur in the midst of daily operation. The problem is represented as a TBN and modeled as a minimum cost flow model with side constraints. Several experiments were carried out to demonstrate the effectiveness of the approach, using data from Continental Airlines. The reported results show that very good solutions are obtained in 3 CPU minutes, on average.

Løve et al. (2002) solved the ARP that included flights delays and cancelation costs using two local search approaches: the steepest ascent local search (SALS) and the iterated local search. The heuristic methods were based on a network formulation, where nodes are either aircraft or flights. Assigning an aircraft to a given flight corresponds to selecting the edge connecting the aircraft and flight for the solution. Any candidate solution is altered by swaps that exchange flights between two aircrafts. With randomly generated data, the SALS approach quickly finds a local optimum (2.11 s on average). Kohl et al. (2007) confirmed the results of Løve et al. (2002) on real-life data. As part of the DESCARTES project, they also presented a crew scheduling solver and described a prototype multipleresource rescheduling decision-support system for a disruption management of aircrafts, flights, and crews.

Dožić (2009) presented a formulation for the ARP which had the objective of minimizing the total delay costs. The model incorporated the following constraints: time-window constraints, aircraft maintenance constraints, aircraft balance constraints, and capacity constraints. A local search heuristic was developed for obtaining a list of feasible solutions ordered according to the objective function value. The heuristic is based on rotation crossing of delayed flights, achieved by removing part of one rotation and adding to another rotation, or by interchanging parts of two rotations. A numerical example for one operational day was conducted for 29 aircraft (nine different aircraft types), assigned to 126 flights. A feasible solution list is obtained in less than $10 \mathrm{~s}$ for selection and implementation by a dispatcher.

Bratu and Barnhart (2006) proposed two optimization models to generate integrated recovery plans for aircraft, 


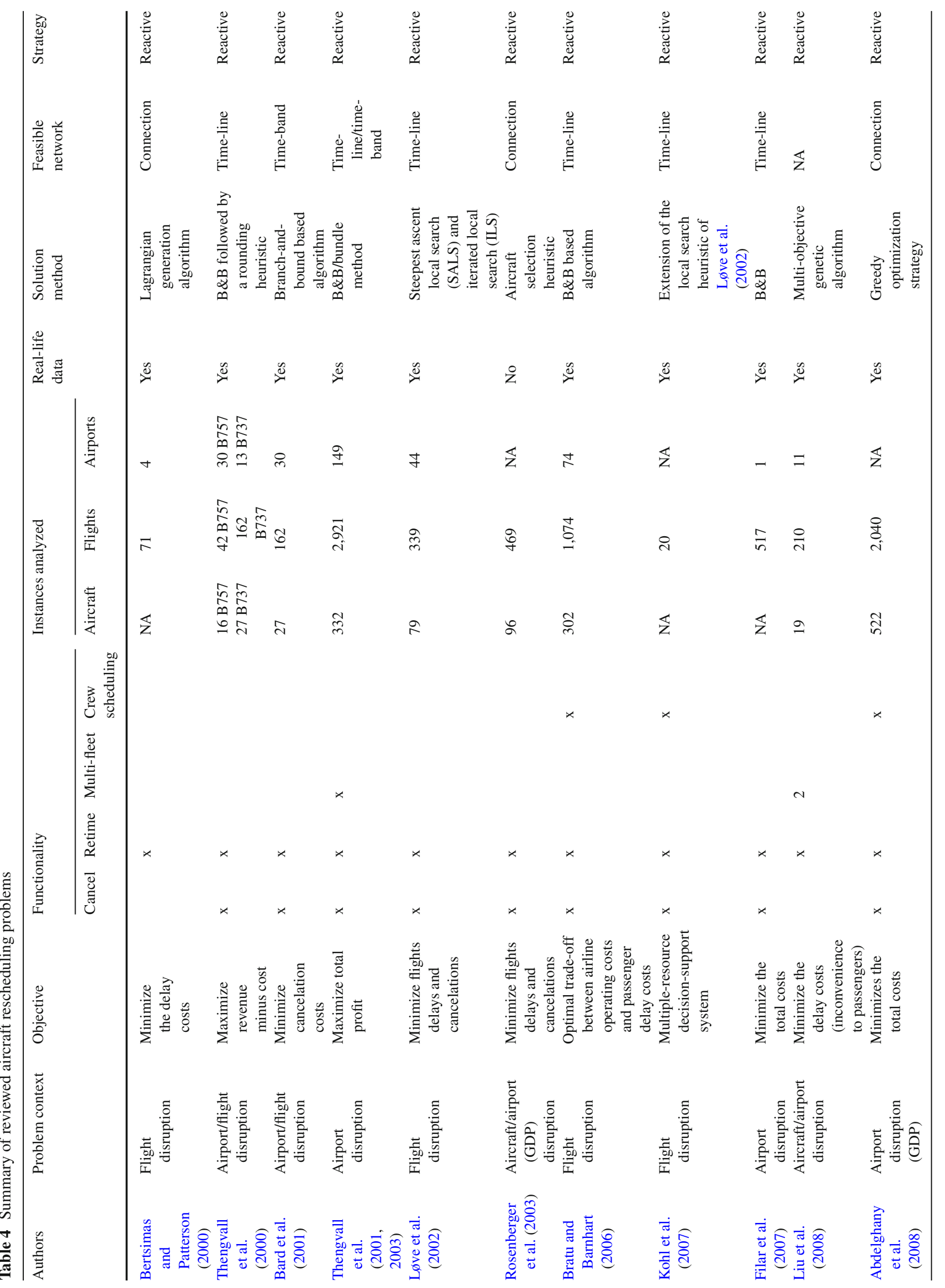




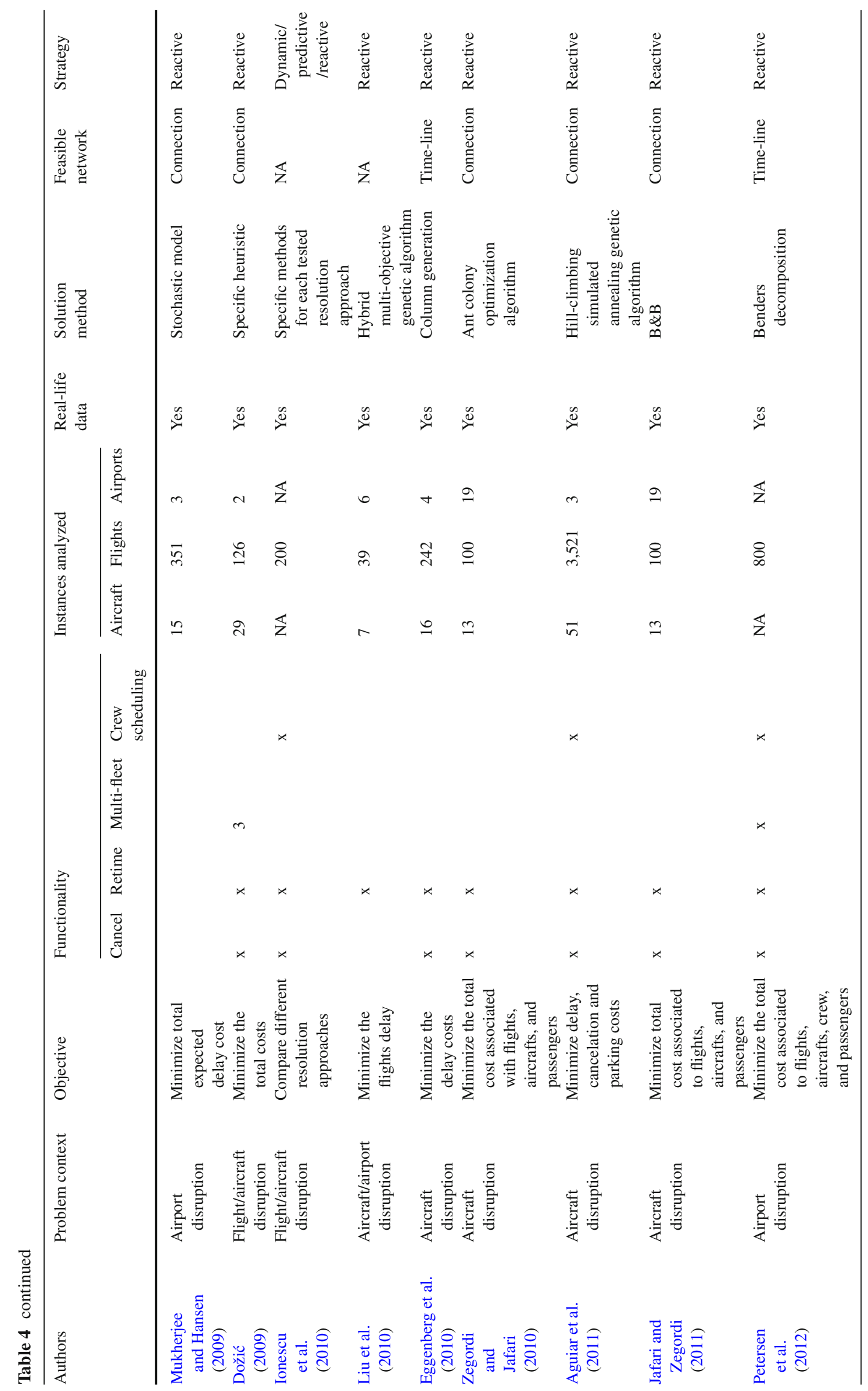


crews, and passengers which allow to delay or cancel flight departures, while ensuring the compliance of crew regulations and aircraft maintenance requirements. Their objective function finds the optimal trade-off between airline operating costs and passenger delay costs. An airline operations control center simulator was developed to evaluate the airline recovery models for real instances of airline operations. Compared to the actual operations data which included actual passenger delays, the models of Bratu and Barnhart reduced the number of disrupted passengers who stayed overnight by $17.2 \%$, and reduced average passenger delay by $5.2 \%$.

Considering the crew connection problem, Aguiar et al. (2011) developed an aircraft recovery approach, taking into account a multi-objective approach that optimizes delays and other costs associated to the use of aircrafts. To solve the ARP, three different meta-heuristics were implemented: hillclimbing, SA, and genetic algorithm. Although all developed heuristics performed well in terms of obtaining acceptable delays through time, the genetic algorithm implementation presented the better results. The crew connecting problem is solved by the solution obtained on the aircraft recovery. To solve it, hill-climbing and SA algorithms were developed and tested using data from TAP Portugal. Simulated annealing presented better results in terms of wages decrease.

Testing another meta-heuristic, Zegordi and Jafari (2010) solved the ARP with an ant colony algorithm, adding considerations about disrupted passengers to the problem. To achieve that, the authors consider aircraft rotations and passengers' itineraries instead of flights. The aircraft recovery is formulated as a MILP in which the objective is to minimize the total cost associated to recovering all flights, aircrafts, and passengers affected by the disruption. The ant colony algorithm is tested on real-world instances obtained from Andersson (2006), and solved the problem with good solutions within few minutes of computation time. This same model is solved in Jafari and Zegordi (2011), using real data instances presented by Andersson and Värbrand (2004). LINGO was employed to solve the problems. However, as the size of the instances increase, the commercial solver could not find solutions in a reasonable amount of time. Four preprocessing steps were then developed to decrease the problem complexity, aiming at reducing the number of integer variables and constraints. The computational time for solving the problem is not mentioned, but the costs are equal to or smaller than those found by the precursor paper for the tested instances.

\subsubsection{Airport disruption}

Thengvall et al. $(2001,2003)$ extended their previous work (Thengvall et al. 2000) to deal with hub closures in a multifleet context. In the 2001 paper, three multi-commodity flow problems were introduced aiming at maximizing the revenue minus costs of disrupted flights. Two models (Models 1 and
2) are based on time-line networks, while the third model (Model 3) is based on a TBN. Model 3 is quicker for shorter closure times, but slower for larger closure times. A bundle algorithm to solve the Lagrangian relaxation of Model 3 is introduced in the 2003 paper. Optimal solutions were found by the bundle method quicker in extensive experiments using data from Continental Airlines.

Filar et al. (2007) studied airport disruptions, and developed a new optimization model called model for adaptive rescheduling of flights in emergencies (MARFE). MARFE modifies schedules for both aircrafts that are still on the ground and for those that are already in the air, constrained by airport capacity levels. MARFE attempts to minimize the sum of delay costs, curfew violation costs, cancelation costs, and diversion costs. MARFE took less than $5 \mathrm{~min}$ to solve problems to optimality for real data obtained from the Sydney airport in Australia, consisting of 517 flights a day, 261 flight arrivals, and 256 departures.

Liu et al. (2008) considered the situation of a 1-h temporary closure of two airports. A multi-objective airline disruption management model was proposed. A genetic algorithm (called MMGA) was developed to generate a timeeffective multi-fleet aircraft recovery aiming at simultaneously minimizing the cost for schedule recovery (considering additional assignment and crew reassignment costs), and passengers' inconvenience. The model formulation considered ground turn-around times, flight connection times, flight swaps, total flight delay time, and a constraint on 30-min maximum delay time from the original schedules. Real flight schedules obtained from a Taiwanese domestic airline were used for evaluation. No running times were given, but the authors indicated that "simulation results demonstrate that the application is capable of presenting high-quality solutions in minutes". In Liu et al. (2010) two soft constraints were added to the previous model for a daily short-haul aircraft schedule recovery problem to minimize delay time variance and the number of delayed flights. Suboptimal solutions (with a $4 \%$ optimality gap) were found in $3.6 \mathrm{~min}$ on average; optimal solutions needed an average of $7.5 \mathrm{~min}$.

Rosenberger et al. (2003) presented an aircraft recovery approach based on the set partitioning problem that considers both aircraft disruptions and station disruptions. The optimization model reschedules legs and reroutes aircraft by minimizing an objective function involving rerouting and cancelation costs. An efficient aircraft selection heuristic was included to identify the subset of aircraft that may be rerouted. The model was validated by simulation experiments considering 500 days of airline operations; three different fleets were analyzed separately.

Abdelghany et al. (2008) developed a decision support tool, DSTAR, for airline schedule recovery during irregular operations aimed at an integrated recovery of aircraft, pilots, and flight attendants. The tool is composed of two integrated 
models, a schedule simulation and a resource assignment optimization model. The first one predicts the list of disrupted flights in the system as function of resource availability and aviation rules. The second one seeks to find the optimal plan of crew and aircraft swapping, reserve utilization, and flight reassignment to recover the projected list of disrupted flights. The recovery horizon was divided into stages, where the simulation model produces the list of disrupted flights for the remaining horizon, and the optimization solver makes minimum cost resource reassignments. The recovery problem at each stage is formulated as a MILP, aiming to minimize the total recovering cost, including resource reassignment cost, total delay cost, and cancelation cost. An experimental investigation using real data indicated that DSTAR has saved approximately $5 \%$ in recovery costs when compared with human schedulers.

Petersen et al. (2012) presented an innovative research, attempting at solving the fully integrated airline recovery problem. The developed optimization-based approach seeks to repair (in an integrated way, rather than sequentially) the flight schedule, aircraft rotations, crew schedule, and passenger itineraries. Given the extreme complexity of the integrated ARP, a routine is included to limit the size of the problem, introducing the notion of disruptable flight set. The routine analyzes the flights that are directly affected by a resource at the airport. Next, these flights are expanded to consider aircraft, crews, and passengers. The disruptable flight set receives all resources that force a delay or cancelation. The solution method, based on a Benders decomposition scheme, has a main objective to optimize passenger delay, consisting in the integration of several complex subproblems, each one corresponding to solve scheduling decisions affecting aircrafts routes and rotations, crew, and passenger itineraries. The authors use data from an American regional company, involving 800 flights and 2 fleets, to evaluate their approach. Solutions are found in reasonable time for several disruption scenarios. Moreover, the quality of the solutions, considering the integrated approach, is improved in comparison with the more traditional sequential method.

\section{Summary and conclusions}

As transportation services become larger and more complex to serve growing demands, the occurrence of severe disruptions grows and their impacts are increasingly costly. A severe disruption can cause significant economic loss and many negative effects on the level and quality of service provided by transportation/logistics companies. The objective of this paper is to present a state-of-the-art review in real-time vehicle schedule recovery modeling and solution methods within train, airline, and road-based transportation. Meeting the challenges of solving RTSVRP quickly and with good quality, considering different vehicle types and disruption causes, provides operations researchers, systems engineers, mathematicians, and computer scientists opportunities for beneficial research and more discoveries.

Considering the flowchart presented in Figs. 4, 5, and 8, it is possible to conclude that the schedule recovery process for air, train, and road-based transportation is similar. The process starts with an analysis of the disruption and its consequence for the initial off-line planning. In general, an experienced staff is responsible for defining whether a disruption is severe enough to ask for a complex recovery of the system, involving reallocation of a set of resources (vehicles, crew, passengers, tracks, cargo, etc.), or a simple recovery heuristic is sufficient to handle the unexpected event. For this initial decision, criteria such as costs and benefits, governmental regulations, duration of the disruption, among others, play very important roles. Clearly, some disruptions do not deserve complex small recovery actions, as follows: traffic delays in the road-based transportation; mishandled luggage and overbooked flight legs in the airline sector; and excessive time for dis/embarking in crowded train stations. The generation of schedule recovery plans is the next step. This process is done in a sequential way, first the vehicle schedule is solved, followed by the crew rescheduling. Next, the impact on passengers is addressed. The recovery process is iterated until a feasible solution is reached for all resources. Considering the diversity of resources involved, disruption causes and contexts, government regulations, and user preferences, the whole recovery problem presents several and conflicting objectives and a very large number of constraints.

Until recently, the generation of recovery plans was made by human experts, employing common sense and past experiences. They are content in producing a very small number of viable plans (sometimes, just one), given the complexity of the task (Clausen et al. 2010). The technological developments on real-time monitoring of mobile units and status of transportation services, and the emergence of new algorithms to solve large optimization models in the last two decades, have resulted in the development of new models and solution methods to cope with real-time recovery in transportation services, especially for the RTVSRP. For two major reasons modeling and solving vehicle recovering problems are more complex than modeling and solving the counterpart planning problems. First, large amounts of data are required to solve the recovery problems and these must be collected in realtime. Second, the recovery problems are quite complex and need to be solved quickly.

The RTVSRP is a large class of problems, considering different vehicle types, several different contexts (mainly in terms of the disruption cause), and many objectives. Although there is a great variety in the way RTVSRP instances are formulated and solved, all reviewed papers follow a similar modeling approach. The problems are initially 
represented using an underlying network structure to model the schedules. In these structures, possible alternatives and some constraints are explicitly represented. Based on these networks, the problems are mathematically formulated and solved using approaches that either decompose the problem into smaller easier-to-solve classical OR problems or use heuristic search methods. Section 2 presents a generic (and didactical) formulation for the RTVSRP. Next, we analyze the underlying networks, mathematical formulation, and solution methods employed to solve the RTVSRP instances for airline and ground transportation, identifying similarities and differences.

Based on Tables 2, 3, and 4, three different network structures were identified in the RTVSRP literature, namely connection, time-line, and time-band. The most commonly used network for this problem is the connection. This is not a surprise, since this structure is also the most used in offline scheduling processes. In the road-base transportation, almost all problems with the exception of Ernst et al. (2007) use this structure. But the problem is directed to recreational rental vehicles rather than to public transportation, as usual in the RTVSRP applied to road-based transportation. In the railway-based transportation, the time-line network appears in some papers (around $18 \%$ of the revised papers). But it is in the airline-based transportation that the alternative networks to connection seem to have an important role (44\% of the revised papers use time-line or time-based networks). Although time-line and TBN are more complex, they explicitly account for time and space simultaneously, while in the connection only space is represented; time is only implicitly represented in the arcs. We speculate that the costs involved in the airline-based transportation demand more precise solutions and better ways to generate recovery plans. Bard et al. (2001) and Thengvall et al. $(2001,2003)$ claim that time-line and TBN, however more complex to be generated, are able to offer these benefits when solving real-world problems, originating mathematical models quicker to solve. In particular, Thengvall et al. (2001) presents a comparison on the use of these networks for the same problem. Time-band performed better when the disruption time was lower. On the other hand, time-line performed better when the disruption time was larger. This can be explained by the greater level of detail demanded by the TBN. As the disruption time increases, the number of components being represented increases exponentially, generating excessive binary variables in the respective mathematical formulation.

The RTVSRP is often modeled using similar approaches as their planning counterpart problems. Several mathematical formulations are based on multi-commodity network flows, set partitioning, and assignment problems. The real-time perspective is represented by including a set of integer (in general, binary) decision variables and side constraints. The VRSP and the ARP present some similarities in the ways their subproblems are formulated, based on classical integer linear programming models such as the network flow problem, the set partitioning problem, and the assignment problem. Specific technical-related constraints, involving track number, track directions, and train types, make the TRP a little different. TRP has been formulated considering event-activity networks, disjunctive programs, and event-driven MILP. As for their computational complexity, both VRSP and ARP have the same complexity, and are proven to be NP-hard problems. Strotmann (2007) proved that TRP is NP-complete.

The solution methods used to solve the RTVSRP are also similar to the ones used for off-line planning problems, such as B\&B, Lagrangian relaxation, column generation, soft computing heuristics, and local-search based heuristics. The solution approaches for VRSP are based on traditional optimization methods developed for the schedule design process; soft computing and local search based heuristics have been neglected. Based on the reviewed literature, the TRP seems to be the most difficult problem to solve in practice, as a consequence of the following aspects: the severe security measures (two trains cannot occupy simultaneously the same segment); the number of resources involved (trains, segments, blocks, and stations); the control of the decision is disperse through different levels; and the generalized impacts of the so-called "snow ball effect". In the TRP, a common solving approach clearly emerged due to its high complexity. The problem is initially relaxed or transformed into a simpler one. This problem is solved using the classical developed methods to handle large integer programming models (Lagrangian relaxation and column generation). Soft computing and local search based heuristics are then developed to improve or to build a viable solution from this initial solution. In the ARP, several different solving methods can be observed. Besides the two approaches previously described for the VRSP and the TRP, metaheuristic approaches have become popular in the last years. The capacity of generating multiple solutions with different characteristics in a very reasonable amount of time (Andersson 2006), even for real-time context, justifies its wide utilization in the ARP, highly dependent on a set of alternative plans as presented in Fig. 8.

The analysis of the literature on the RTVSRP reveals some additional interesting facts. The first is that the literature on VRSP is scant compared with train and aircraft recovery literature. This aspect was highlighted by Daduna and Paixão (1995) and remains true today. Disruptions and realtime recovery for buses and trucks are topics that need to be further explored, mainly on the application of new solution methods. A good starting point for some new approaches for VRSP would be to apply some of the methods developed for ARP, given the similarities of the problems.

Another observation based on the literature review is the small number of papers related to major technical failures or severe accidents in the RTVSRP. The majority 
of the reviewed papers consider delays as the only cause of disruption. Exceptions are $\mathrm{Li}$ et al. (2007a,b, 2008, 2009) for the VRSP, and Eggenberg et al. (2010) for the ARP. Although not as common as delays, vehicle mechanical problems and accidents have higher average durations among vehicle disruptions (Acuna-Agost et al. 2011). Vehicle breakdowns/accidents require the consideration of additional issues, such as the need to serve the passengers/cargo involved in the disruption. Solution methods that solve for only delays, in general, cannot be directly applied to mechanical failures and accidents. Furthermore, new robust strategies evolve when technical failures are considered, such as the necessity of keeping spare vehicles parked in strategic locations to recover the passengers/cargo.

Few papers integrate vehicle, crew, and passenger recovery. For the VRSP, Huisman and Wagelmans (2006) analyzed these aspects in an integrated way, but the developed solution method required high computation times. For the TRP, Sato et al. (2009) integrated the TRP and crew rescheduling problem based on a network flow model. The situation is slightly better in the ARP, where Kohl et al. (2007) and Abdelghany et al. (2008) integrated the aircraft and crew scheduling, while Bratu and Barnhart (2006) and Petersen et al. (2012) integrated aircraft, crew, and passenger recovery. The solutions provided in the ARP context show the great potential of integrated approaches for obtaining global solutions for disruption management. In general, the optimum solutions of the VRSP/ARP/TRP could be far from the optimum integrated solution for a recovery.

\section{Implications for future research}

The RTVSRP provides OR scientists with an interesting and challenging field of study. There is still much work to be done and much to discover on the subject. The following subjects are promising areas for future research.

The RTVSRP can also involve multiple and conflicting objectives in regulated environments. Although several papers formulate RTVSRPs to include costs and delays in their objective functions, very few papers use multiobjective optimization for solving the problem. Among papers reviewed, Liu et al. $(2008,2010)$ are well-received exceptions, adopting a multi-objective optimization approach for the ARP. As multiple, often conflicting, objectives arise naturally in the RTVSRP, multi-objective approaches, should receive greater attention in the near future.

There is a clear trend in our review of solving the RTVRSP to improve the operational efficiency of the transportation service provider. Very few papers include or put emphasis on passengers' wishes and objectives in their formulations. Nielsen $(2008,2011)$ and Berger et al. (2011a) in the TRP, and Liu et al. (2008) and Petersen et al. (2012) in the ARP are recent developments in this direction. The problem is quite complex and several aspects such as passengers' behavior toward serious disruptions was not properly addressed. We are quite sure that passenger disruption management will be a very hot research topic in the near future.

Robust and real-time scheduling are highly correlated themes of research. However, they have been treated as isolated and completely independent problems. We believe that the integration of these approaches into decision support systems can offer very effective tools to minimize the effects of light as well as severe disruptions on daily operations of transportation services. A promising field of study related with robust scheduling is to introduce stability as a performance measure index to analyze and evaluate rescheduling in transportation.

The use of TLN is observed in the three types of transportation services discussed in this paper, being more common in train and ARPs. Extensions of this kind of network, called time-space networks, were developed for the off-line scheduling processes. The time-space networks was first suggested by Hane et al. (1995) and Clarke et al. (1996) for solving an airline scheduling problem. In a time-space network, a node represents a specific location at a particular time while an arc corresponds to a transition in time, representing actions that can be performed by a vehicle. The dynamics within a location is represented by using a time-line that connects all possible start and end events that can occur in this location. Pull-in/out arcs to/from stations or depots, and deadheading arcs are added to model schedules. Additionally, these networks are modeled in a cycle, which forces the solution to be a circulation flow through the network. Kliewer et al. (2006) adapted them to solve the bus scheduling problem. Following, Steinzen (2007) and Steinzen et al. (2010) expanded these networks to be used in an integrated multiple depot vehicle and crew scheduling problem. They also developed techniques that lead to a drastic reduction of deadheading arcs, reducing the network size in comparison to connection networks. The application of time-space networks to the RTVRSP could generate very friendly networks, allowing the development of innovative and less complex models with quicker solution methods.

In the last decade, constraint programming (CP) has been widely applied to scheduling problems (Lombardi and Milano 2012). CP has as its major principle the "deduction of additional constraints from existing ones by logical reasoning" (Baptiste et al. 2001). Although CP is aligned of how RTVRSP is solved in practice, we could not find any application of $\mathrm{CP}$ to this problem as $\mathrm{CP}$ has proven very successful in scheduling, offering significant advantages such as fast program development support, economic program maintenance, and efficient run time performance (Wallace 1996), we believe that $\mathrm{CP}$ can be applied toward the development of good and quick solution methods to the RTVRSP. 
Acknowledgments We are indebted to the anonymous referees for very useful comments and criticism. This work was partially funded by Conselho Nacional de Desenvolvimento Científico e Tecnológico (CNPq), Brazil.

\section{References}

Abdelghany, K., Abdelghany, A., \& Ekollu, G. (2008). An integrated decision support tool for airlines schedule recovery during irregular operations. European Journal of Operational Research, 185(2), 825848.

Acuna-Agost, R., Michelon, P., Feillet, D., \& Gueye, S. (2011). A MILP-based local search method for the railway rescheduling problem. Networks, 57, 69-86.

Ageeva, Y. (2000). Approaches to incorporating robustness into airline scheduling. Master's thesis. Massachusetts Institute of Technology, Cambridge.

Aguiar, B., Torres, J., \& Castro, A. J. M. (2011). Operational problems recovery in airlines-a specialized methodologies approach. Lecture Notes in Computer Science, 7026, 83-97.

Ahuja, R. K., Möhring, R. H., \& Zaroliagis, C. D. (2009). Robust and online large scale optimization: Models and techniques for transportation systems. Berlin: Springer.

Amberg, B., Amberg, B., \& Kliewer, N. (2012). Increasing delaytolerance of vehicle and crew schedules in public transport by sequential, partial-integrated and integrated approaches. ProcediaSocial and Behavioral Sciences, 20, 292-301.

Andersson, T. (2006). Solving the flight perturbation problem with meta heuristics. Journal of Heuristics, 12, 37-53.

Andersson, T., \& Värbrand, P. (2004). The flight perturbation problem. Transportation Planning and Technology, 27, 91-117.

Almodóvar, M., \& García-Ródenas, R. (2013). On-line reschedule optimization for passenger railways in case of emergencies. Computers \& Operations Research, 40(3), 725-736.

Argüello, M., Bard, J., \& Yu, G. (1997). A GRASP for aircraft routing in response to groundings and delays. Journal of Combinatorial Optimization, 5, 211-228.

Arora, S., \& Barak, B. (2009). Computational complexity: A modern approach. Cambridge: Cambridge University Press.

Babić, O., Kalić, M., Pavković, G., Dožić, S., \& Čangalović, M. (2010). Heuristic approach to the airline schedule disturbances problem. Transportation Planning and Technology, 33(3), 257-280.

Bard, J., Yu, G., \& Argüello, M. (2001). Optimizing aircraft routings in response to groundings and delays. IIE Transactions, 33, 931-947.

Ball, M., Barnhart, C., Nemhauser, G., \& Odoni, A. (2007). Air transportation: Irregular operations and control. In C. Branhart \& G. Laporte (Eds.), Handbook in OR \& MS (Vol. 14, pp. 1-67). Amsterdam: Elsevier.

Baptiste, P., Pape, C., \& Nuyten, W. (2001). Constraint-based scheduling: Applying constraint programming to scheduling problems. New York: Springer-Verlag LLC.

Berger, A., Blaar, C., Gebhardt, A., Müller-Hannemann, M., \& Schnee, M. (2011a). Passenger flow-oriented train disposition. Lecture Notes in Computer Science, 6942, 227-238.

Berger, A., Hoffmann, R., Lorenz, U., \& Stiller, S. (2011b). Online railway delay management: Hardness, simulation and computation. Simulation, 87(7), 616-629.

Bertsimas, D., \& Patterson, S. S. (2000). The traffic flow management rerouting problem in air traffic control: A dynamic network flow approach. Transportation Science, 34(3), 239-255.

Bisaillon, S., Cordeau, J.-F., Laporte, G., \& Pasin, F. (2011). A large neighborhood search heuristic for the aircraft and passenger recovery problem. 4OR, A Quarterly Journal of Operations Research, 9(2), 139-157.
Bratu, S., \& Barnhart, C. (2006). Flight operations recovery: New approaches considering passenger recovery. Journal of Scheduling, 9, 279-298.

Brazilian Civil Aviation Agency. (2010). Anuário do Transporte Aéreo 2010 (1st ed.). http://www2.anac.gov.br/estatistica/anuarios. asp. Accessed 10 Apr 2010.

Borndörfer, R., Dovica, I., Nowak, I., \& Schickinger, T. (2009). Robust tail assignment. ZIB, Report 10-08.

Bunte, S., \& Kliewer, N. (2009). An overview on vehicle scheduling models in public transport. Public Transport, 1(4), 299-317.

Caprara, A., Galli, L., Kroon, L., Maróti, G., \& Toth, P. (2010). Robust train routing and online re-scheduling. In T. Erlebach \& M. Lübbecke (Eds.), 10th Workshop on algorithmic approaches for transportation modelling, optimization, and systems (ATMOS'10) (pp. 24-33). Dagstuhl.

Cao, J.-M., \& Kanafani, A. (1997a). A real-time decision support for integration of airline flight cancellation and delays, part I: Mathematical formulation. Transportation Planning and Technology, 20, 183-199.

Cao, J.-M., \& Kanafani, A. (1997b). A real-time decision support for integration of airline flight cancellation and delays, part II: Algorithm and computation. Transportation Planning and Technology, 20, 201217.

Christiansen, M., Fagerholt, K., \& Ronen, D. (2004). Ship routing and scheduling: Status and perspectives. Transportation Science, 38(1), $1-18$.

Clarke, L. W., Hane, C. A., Johnson, E. L., \& Nemhauser, G. L. (1996). Maintenance and crew considerations in fleet assignment. Transportation Science, 30(3), 249-260.

Clausen, J., Larsen, A., Larsen, J., \& Rezanova, N. J. (2010). Disruption management in the airline industry: Concepts, models and methods. Computers \& Operations Research, 37(5), 809-821.

Cordeau, J.-F., Toth, P., \& Vigo, D. (1998). A survey of optimization models for train routing and scheduling. Transportation Science, 32(4), 380-404.

Corman, F., D’Ariano, A., Pacciarelli, D., \& Pranzo, M. (2010). A tabu search algorithm for rerouting trains during rail operations. Transportation Research Part B: Methodological, 44(1), 175192.

D'Ariano, A. (2008). Improving real-time train dispatching: Models, algorithms and applications. Ph.D thesis. Department of Transport \& Planning, Faculty of Civil Engineering and Geosciences, Delft University of Technology, The Netherlands.

D’Ariano, A. D., \& Pranzo, M. (2009). An advanced real-time train dispatching system for minimizing the propagation of delays in a dispatching area under severe disturbances. Networks and Spatial Economics, 9(1), 63-84.

D’Ariano, A., Corman, F., Pacciarelli, D., \& Pranzo, M. (2008). Reordering and local rerouting strategies to manage train traffic in real-time. Transportation Science, 42(4), 405-419.

D'Ariano, A., Pacciarelli, D., \& Pranzo, M. (2007a). A branch and bound algorithm for scheduling trains in a railway network. European Journal of Operational Research, 183(2), 643-657.

D'Ariano, A., Pranzo, M., \& Hansen, I. A. (2007b). Conflict resolution and train speed coordination for solving real-time timetable perturbations. IEEE Transactions on Intelligent Transportation Systems, 8(2), 208-222.

Daduna, J. R., \& Paixão, J. M. (1995). Vehicle scheduling for public mass transit-an overview. In Proceedings of the 6th international conference on computer-aided scheduling of public transport, Boston, MA, pp. 76-90.

Dirksen, B. J. (2011). Disruption management in liner shipping. Master's dissertation. Technical University of Denmark.

Dožić, S., Kalić, M., Babić, O., \& Čangalović, M. (2009). Heuristic approach to the airline schedule disturbances problem: Multi-fleet 
case. In Multidisciplinary international conference on scheduling: Theory and applications (MISTA 2009). Dublin, Ireland.

Dück, V., Ionescu, L., Kliewer, N., \& Suhl, L. (2012). Increasing stability of crew and aircraft schedules. Transportation Research Part C: Emerging Technologies, 20, 47-61.

Ernst, A. T., Horn, M., Krishnamoorthy, M., Kilby, P., Degenhardt, P., \& Moran, M. (2007). Static and dynamic order scheduling for recreational rental vehicles at tourism holdings limited. Interfaces, 37(4), 334-341.

Eggenberg, N., Salani, M., \& Bierlaire, M. (2010). Constraint-specific recovery network for solving airline recovery problems. Computers \& Operations Research, 37(6), 1014-1026.

Fekete, S. P., Kroeller, A., Lorek, M. \& Pfetsch, M. E. (2011). Disruption management with rescheduling of trips and vehicle circulations. In Proceedings of the 5th ASME/ASCE/IEEE Joint Rail Conference. Pueblo, Colorado, USA.

Filar, J. A., Manyem, P., Panton, D. M., \& White, K. (2007). A model for adaptive rescheduling of flights in emergencies (MARFE). Journal of Industrial and Management Optimization, 3(2), 335-356.

Fischetti, M., Salvagnin, D., \& Zanette, A. (2009). Fast approaches to improve the robustness of a railway timetable. Transportation Science, 43(3), 321-335.

Freling, R., Wagelmans, A. P. M., \& Paixão, J. M. P. (2001). Models and algorithms for single-depot vehicle scheduling. Transportation Science, 35(2), 165-180.

García-Ródenas, R., Almodóvar, M., \& Parreño, F. (2009). Heuristic algorithm for coordination in public transport under disruptions. Lecture Notes in Computer Science, 5484, 808-817.

Guarino, J., \& Firestine, T. (2010). Effects of the February 2010 snowstorms on airline performance. Special Report-021, Rita Bureau of Transportation Statistics, US Department of Transportation.

Hane, C. A., Barnhart, C., Johnson, E. L., Marsten, R. E., Nemhauser, G. L., \& Sigismondi, G. (1995). The fleet assignment problem: Solving a large-scale integer program. Mathematical Programming, 70, 211 232.

Herroelen, W., \& Leus, R. (2004). The construction of stable project baseline schedules. European Journal of Operational Research, 156, $550-565$.

Huisman, D., \& Wagelmans, A. (2006). A solution approach for dynamic vehicle and crew scheduling. European Journal of Operational Research, 172(2), 453-471.

Huisman, D., Freling, R., \& Wagelmans, A. P. M. (2004). A robust solution approach to the dynamic vehicle scheduling problem. Transportation Science, 38(4), 447-458.

Ionescu, L., Kliewer, N., \& Schramme, T. (2010). A comparison of recovery strategies for crew and aircraft schedules. In B. Hu, K. Morasch, S. Pickl, \& M. Siegle (Eds.), Operations research proceedings 2010 (pp. 269-274). Berlin: Springer.

Jafari, N., \& Zegordi, S. H. (2011). Simultaneous recovery model for aircraft and passengers. Journal of the Franklin Institute, 348, 16381655.

Jarrah, A., Yu, G., Krishnamurthy, N., \& Rakshit, A. (1993). A decision support framework for airline flight cancellation and delays. Transportation Science, 27, 266-280.

Jespersen-Groth, J., Potthoff, D., Clausen, J., Huisman, D., Kroon, L., Gábor, M., et al. (2009). Disruption management in passenger railway transportation. In R. K. Ahuja (Ed.), Robust and online largescale optimization (pp. 399-421). Berlin: Springer-Verlag.

Kliewer, N., Mellouli, T., \& Suhl, L. (2006). A time-space network based exact optimization model for multi-depot bus scheduling. European Journal of Operational Research, 175(3), 16161627.

Kohl, N., Larsen, A., Larsen, J., Ross, A., \& Tiourine, S. (2007). Airline disruption management-perspectives, experiences and outlook. Journal of Air Transport Management, 13(3), 149-162.
Kramkowski, S., Kliewer, N., \& Meier, C. (2009). Heuristic methods for increasing delay-tolerance of vehicle schedules in public bus transport. In Proceedings of the metaheuristic international conference VIII. Hamburg, Germany.

Kroon, L. G. \& Huisman, D. (2011). Algorithmic support for disruption management at Netherlands Railways. Econometric Institute Report. EI 2011-06. Econometric Institute, Erasmus University, Rotterdam.

Lan, S., Clarke, J.-P., \& Barnhart, C. (2006). Planning for robust airline operations: Optimizing aircraft routings and flight departure times to minimize passenger disruptions. Transportation Science, 40(1), $15-28$.

Lee, L. H., Lee, U. C., \& Tan, Y. P. (2007). A multi-objective genetic algorithm for robust flight scheduling using simulation. European Journal of Operational Research, 177, 1948-1968.

Leus, R., \& Herroelen, W. (2005). The complexity of machine scheduling for stability with a single disrupted job. Operations Research Letters, 33(2), 151-156.

Li, J. Q., Borenstein, D., \& Mirchandani, P. B. (2008). Truck schedule recovery for solid waste collection in Porto Alegre. International Transactions in Operational Research, 15, 565-582.

Li, J. Q., Mirchandani, P. B., \& Borenstein, D. (2004). Parallel auction algorithm for bus rescheduling. In Proceedings of ninth international conference on computer-aided scheduling of public transport. San Diego, CA.

Li, J. Q., Mirchandani, P. B., \& Borenstein, D. (2007a). The vehicle rescheduling problem: Model and algorithms. Networks, 50(3), 211229.

Li, J., Borenstein, D., \& Mirchandani, P. B. (2007b). A decision support system for the single-depot vehicle rescheduling problem. Computers \& Operations Research, 34(4), 1008-1032.

Li, J. Q., Mirchandani, P. B., \& Borenstein, D. (2009). A Lagrangian heuristic for the real-time vehicle rescheduling problem. Transportation Research Part E: Logistics and Transportation Review, 45(3), 419-433.

Liu, T. K., Chen, C. H., \& Chou, J. H. (2010). Optimization of short-haul aircraft schedule recovery problems using a hybrid multiobjective genetic algorithm. Expert Systems with Applications, 37(3), 23072315.

Liu, T. K., Jeng, C. R., \& Chang, Y. H. (2008). Disruption management of an inequality-based multi-fleet airline schedule by a multiobjective genetic algorithm. Transportation Planning and Technology, 31(6), 613-639.

Lombardi, M., \& Milano, M. (2012). Optimal methods for resource allocation and scheduling: A cross disciplinary survey. Constraints, 17(1), 51-85.

Løve, M., Sørensen, K. R., Larsen, J., \& Clausen, J. (2002). Disruption management for an airline-rescheduling of aircraft. In EvoWorkshops 2002, LNCS 2279 (pp. 315-324). Berlin, Heidelberg: SpringerVerlag.

Luethi, M., Medeossi, G., \& Nash, A. (2009). Structure and simulation evaluation of an integrated real-time rescheduling system for railway networks. Networks and Spatial Economics, 9(1), 103-121.

Mathaisel, D. (1996). Decision support for airline system operations control and irregular operations. Computers and Operations Research, 23, 1083-1098.

Mascis, A., \& Pacciarelli, D. (2000). Machine scheduling via alternative graphs. Working paper, Dipartamento di Informatica e Automazione, Università degli Studi Roma, Tré, Italy.

Mascis, A., \& Pacciarelli, D. (2002). Job shop scheduling with blocking and no-wait constraints. European Journal of Operational Research, 143(3), 498-517.

Meng, X., Jia, L., \& Qin, Y. (2010). Train timetable optimizing and rescheduling based on improved particle swarm algorithm. Transportation Research Record: Journal of the Transportation Research Board, 2197(1), 71-79. 
Mukherjee, A., \& Hansen, M. (2009). A dynamic rerouting model for air traffic flow management. Transportation Research Part B: Methodological, 43(1), 159-171.

Nielsen, L. K. (2008). A decision support framework for rolling stock rescheduling. Technical report ARRIVAL-TR-0158.

Nielsen, L. K. (2011). Rolling stock rescheduling in passenger railways-applications in short-term planning and in disruption management. $\mathrm{PhD}$ thesis. Erasmus University, Rotterdam, The Netherlands.

Norio, T., Yoshiaki, T., Noriyuki, T., Chikara, H., \& Kunimitsu, M. (2005). Train rescheduling algorithm which minimizes passengers' dissatisfaction. Lecture Notes in Computer Science, 3533, 829-838.

Petersen, J. D., Gustaf, S., Johnson, E. L., Clarke, J. P., \& Shebalov, S. (2012). An optimization approach to airline integrated recovery system. Transportation Science, 46(4), 482-500.

Potthoff, D., Huisman, D., \& Desaulniers, G. (2010). Column generation with dynamic duty selection for railway crew rescheduling. Transportation Science, 44(4), 493-505.

Raheja, A. S., \& Subramaniam, V. (2002). Reactive recovery of job shop schedules-a review. International Journal of Advanced Manufacturing Technology, 19, 756-763.

Rangsaritratsamee, R., Ferrel, W. G., \& Kurtz, M. B. (2004). Dynamic scheduling that simultaneously considers efficiency and stability. Computers \& Industrial Engineering, 46, 1-15.

Rosenberger, J. M., Johnson, E. L., \& Nemhauser, G. L. (2003). Rerouting aircraft for airline recovery. Transportation Science, 37(4), 408421.

Sahin, I. (1999). Railway traffic control and train scheduling based on inter-train conflict management. Transportation Reseacrh: Part B, $33,511-534$.

Sato, K., \& Fukumura, N. (2012). Real-time freight locomotive rescheduling and uncovered train detection during disruption. European Journal of Operational Research, 221, 636-648.

Sato, T., Shuichiro, S., Morita, T., Ueki, N. \& Murata, T. (2009). Crew and vehicle rescheduling based on a network flow model and its application to a railway train operation. IAENG International Journal of Applied Mathematics, 39(3), IJAM_39_2.

Sato, T., Tomiyama, T., Morita, T. \& Murata, T. (2010). Lagrangian relaxation method for network flow modeled crew and vehicle rescheduling. In Proceedings of the 2nd international conference on advanced computer control (ICACC) (pp. 403-408).

Serafini, P., \& Ukovich, W. (1989). A mathematical model for periodic scheduling problems. SIAM Journal on Discrete Mathematics, 2(4), $550-581$.

Steinzen, I. (2007). Topics in integrated vehicle and crew scheduling in public transit. $\mathrm{PhD}$ thesis. University of Paderborn, Germany.

Steinzen, I., Gintner, V., Suhl, L., \& Kliewer, N. (2010). A time-space network approach for the integrated vehicle-and crew-scheduling problem with multiple depots. Transportation Science, 44(3), 367382.

Strotmann, C. (2007). Railway scheduling problems and their decomposition. Ph.D. thesis. Universität Osnabrück, Germany.
SunTran (2011, July). SunTran Monthly report.

Swedish Transport Administration. (2011). Annual Report 2011. http:// publikationswebbutik.vv.se/upload/6814/2012_083_swedish_ transport_administration_annual_report_2011.pdf. Accessed 10 Apr 2010.

Teodorovic, D., \& Guberinic, S. (1984). Optimal dispatching strategy on an airline network after a schedule perturbation. European Journal of the Operational Research, 15, 178-182.

Thengvall, B., Bard, J. F., \& Yu, G. (2000). Balancing user preferences for aircraft schedule recovery during irregular operations. IIE Transactions, 32, 181-193.

Thengvall, B., Bard, J. F., \& Yu, G. (2003). A bundle algorithm approach for the aircraft schedule recovery problem. Transportation Science, 37, 392-407.

Thengvall, B., Yu, G., \& Bard, J. F. (2001). Multiple fleet aircraft schedule recovery following hub closures. Transportation Research Part A, 35, 289-308.

Törnquist, J. (2006). Computer-based decision support for railway traffic scheduling and dispatching: A review of models and algorithms. In Proceedings of the 5th workshop on algorithmic methods and models for optimization of railways (ATMOS 2005).

Tornquist, J., \& Persson, J. (2007). N-tracked railway traffic rescheduling during disturbances. Transportation Research Part B: Methodological, 41(3), 342-362.

Veelenturf, L. P., Potthoff, D., Huisman, D., \& Kroon, L. G. (2012). Railway crew rescheduling with retiming. Transportation Research Part C: Emerging Technologies, 20(1), 95-110.

Wallace, M. (1996). Practical applications of constraint programming. Constraints: An International Journal, 1, 139-168.

Weide, O., Ryan, D., \& Ehrgott, M. (2009). An iterative approach to robust and integrated aircraft routing and crew scheduling. Computers \& Operations Research, 37, 833-844.

Yan, S., \& Yang, D.-H. (1996). A decision support framework for handling schedule perturbations. Transportation Research Part B: Methodological, 30, 405-419.

Luo, S., \& Yu, G. (1997). On the airline schedule perturbation problem caused by the ground delay program. Transportation Science, 31(4), 298-311.

Zegordi, S. H., \& Jafari, N. (2010). Solving the airline recovery problem by using ant colony optimization. International Journal of Industrial Engineering \& Production Research, 21(3), 121-128.

Zwaneveld, P. J., Kroon, L. G., Romejinn, H. E., Salomon, M., DauzerePeres, S., van Hoesel, C. P. M., et al. (1996). Routing trains through railway stations: Model formulation and algorithm. Transportation Science, 30, 181-194.

Zwaneveld, P. J., Kroon, L. G., \& van Hoesel, C. P. M. (2001). Routing trains through a railway station based on a node packing model. European Journal of Operations Research, 128, 14-33. 